\title{
The Pro-Oncogenic Adaptor CIN85 Acts as an Inhibitory \\ Binding Partner of Hypoxia-Inducible Factor Prolyl Hydroxylase 2
}

\section{Kozlova, Nina}

2019-08-15

Kozlova, N, Mennerich, D , Samoylenko , A, Dimova , E Y, Koivunen , P , Biterova , E , Richter , K, Hassinen , A , Kellokumpu , S , Manninen , A, Minalainen , I, Glumoff , V , Ruddock , L , Drobot , L B \& Kietzmann , T 2019 , ' The Pro-Oncogenic Adaptor CIN85 Acts as an Inhibitory Binding Partner of Hypoxia-Inducible Factor Prolyl Hydroxylase 2 ' , Cancer Research , vol. 79 , no. 16 , pp. 4042-4056 . https://doi.org/10.1158/0008-5472.CAN-18-3852

http://hdl.handle.net/10138/306393

https://doi.org/10.1158/0008-5472.CAN-18-3852

publishedVersion

Downloaded from Helda, University of Helsinki institutional repository.

This is an electronic reprint of the original article.

This reprint may differ from the original in pagination and typographic detail.

Please cite the original version. 


\section{The Pro-Oncogenic Adaptor CIN85 Acts as an Inhibitory Binding Partner of Hypoxia-Inducible Factor Prolyl Hydroxylase 2}

Nina Kozlova,2, Daniela Mennerich², Anatoly Samoylenko ${ }^{2,3}$, Elitsa Y. Dimova ${ }^{2,3}$, Peppi Koivunen $^{2,3}$, Ekaterina Biterova ${ }^{2}$, Kati Richter ${ }^{2}$, Antti Hassinen ${ }^{4}$, Sakari Kellokumpu ${ }^{2}$, Aki Manninen ${ }^{3,5}$, Ilkka Miinalainen ${ }^{5}$, Virpi Glumoff ${ }^{6}$, Lloyd Ruddock ${ }^{2}$, Lyudmyla Borysivna Drobot ${ }^{7}$, and Thomas Kietzmann ${ }^{2,5}$

\section{Abstract}

The EGFR adaptor protein, CIN85, has been shown to promote breast cancer malignancy and hypoxiainducible factor (HIF) stability. However, the mechanisms underlying cancer promotion remain ill defined. Here we show that CIN85 is a novel binding partner of the main HIF-prolyl hydroxylase, PHD2, but not of PHD1 or PHD3. Mechanistically, the N-terminal SRC homology 3 domains of CIN85 interacted with the proline-arginine-rich region within the $\mathrm{N}$-terminus of PHD2, thereby inhibiting PHD2 activity and HIF degradation. This activity is essential in vivo, as specific loss of the CIN85-PHD2 interaction in CRISPR/Cas9edited cells affected growth and migration properties, as well as tumor growth in mice. Overall, we discovered a previously unrecognized tumor growth checkpoint that is regulated by CIN85-PHD2 and uncovered an essential survival function in tumor cells by linking growth factor adaptors with hypoxia signaling.

Significance: This study provides unprecedented evidence for an oxygen-independent mechanism of PHD2 regulation that has important implications in cancer cell survival.

Graphical Abstract: http://cancerres.aacrjournals.org/ content/canres/79/16/4042/F1.large.jpg.

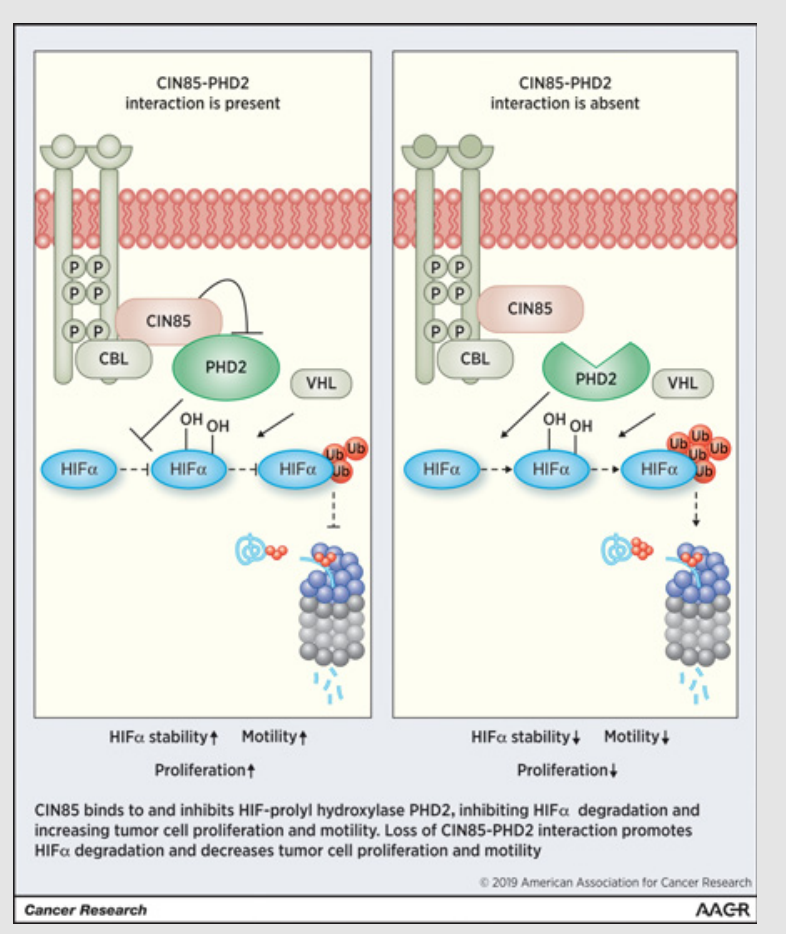

promotors of malignant transformation, cell proliferation, invasion, and motility (2). Although several mechanisms contribute to the regulation of $\mathrm{HIF} \alpha$ expression (3), regulation of HIF stability by the family of HIF-prolyl hydroxylases
${ }^{1}$ Cancer Center at Beth Israel Deaconess Medical Center and Harvard Medical School, Boston, Massachusetts. ${ }^{2}$ Faculty of Biochemistry and Molecular Medicine and Biocenter Oulu, University of Oulu, Oulu, Finland. ${ }^{3}$ Center for Cell-Matrix Research, Faculty of Biochemistry and Molecular Medicine, University of Oulu, Oulu, Finland. ${ }^{4}$ Institute for Molecular Medicine Finland (FIMM), Helsinki, Finland. ${ }^{5}$ Biocenter Oulu, University of Oulu, Oulu, Finland. ${ }^{6}$ The Research Unit of Biomedicine, University of Oulu, Oulu, Finland. ${ }^{7}$ Laboratory of Cell Signaling, Palladin Institute of Biochemistry, National Academy of Sciences of Ukraine, Kyiv, Ukraine.

Note: Supplementary data for this article are available at Cancer Research Online (http://cancerres.aacrjournals.org/).
N. Kozlova and D. Mennerich contributed equally to this article.

Corresponding Author: Thomas Kietzmann, University of Oulu and Biocenter Oulu, Oulu 90230, Finland. Phone: 3582-9448-7714; Fax: 358-8553-1141; E-mail: thomas.kietzmann@oulu.fi

Cancer Res 2019;79:4042-56

doi: 10.1158/0008-5472.CAN-18-3852

C2019 American Association for Cancer Research 
(PHD) is reported to be of major importance. Under normoxic conditions, PHDs hydroxylate proline residues in HIF1 $\alpha$ and HIF2 $\alpha$ (4). This allows further recruitment of an E3-ubiquitin ligase complex containing the von-Hippel Lindau protein (VHL; ref. 5), which results in the proteasomal degradation of HIF1 $\alpha$ and HIF2 $\alpha$.

The prooncogenic adaptor protein, CIN85, is a multi-modular scaffold protein, able of mediating various molecular interactions. Apart from being involved in downregulation of receptor tyrosine kinases such as EGFR (6), ErbB2/Her2 (7), and hepatocyte growth factor receptor (MET; ref. 8), CIN85 also affects apoptosis, adhesion (9), and invasion (10). Earlier findings, including our own, showed that CIN85 promotes development of various cancers including breast cancer and displays highest levels in the most hypoxic areas of tumor tissues, which also usually display high HIF $\alpha$ levels $(11,12)$. In addition, we could previously show that CIN85 appears to induce HIF1 $\alpha$ stability via a so far unknown mechanism (13).

Thus, we hypothesized that CIN85 may affect HIF $\alpha$ stability by affecting the HIF-PHDs. Accordingly, we examined the potential involvement of PHDs in CIN85-mediated HIF $\alpha$ stability in a more mechanistic manner. Our current study shows that CIN85 is a novel binding partner of the main HIF-hydroxylase PHD2. We further defined that the three SRC homology 3 (SH3) domains of CIN85 and the proline-arginine (PR)-rich area within the amino acids 77-100 in the N-terminus of PHD2 are of major importance for the CIN85-PHD2 complex formation. In addition, we utilized a CRISPR/Cas9-mediated EGLN1 (PHD2) gene editing approach to unravel the impact of the CIN85-PHD2 interaction on HIFo stability and cellular behavior in triple-negative breast cancer cells, that is, cells that do not express estrogen receptor, progesterone receptor, and HER2/neu and therefore model the malignant breast cancer growth. The abrogation of PHD2-CIN85 complex formation in the CRISPR/Cas9-edited cells resulted in higher PHD2 activity and subsequently lower HIF1 $\alpha$ and HIF2 $\alpha$ levels, as well as in a less malignant cellular phenotype. Together, we show that CIN85 acts as a novel binding partner of PHD2, which can prevent prolyl-hydroxylation and degradation of HIF $\alpha$ subunits, thereby promoting HIF $\alpha$ stability and breast cancer malignancy.

\section{Materials and Methods}

\section{Chemicals}

All biochemicals and enzymes were of analytic grade and were purchased from commercial suppliers. EGF was from SigmaAldrich, restriction enzymes were from Thermo Fisher Scientific, and FG-4592 was from Cayman Chemical.

\section{Cell culture}

Human embryonic kidney 293 (HEK-293, \# CRL-1573) human breast carcinoma cell lines [MDA-MB-231 (\#HTB-26) Hs578T (\#HTB-126), and BT-549 (\#HTB-122)] were authenticated and purchased from ATCC. All cell lines were tested Mycoplasma negative by using the MycoAlert Detection Kit (Lonza). In all experiments the number of cell passages used was below 10. HEK293, MDA-MB-231, and Hs578TH were maintained in DMEM supplemented with $10 \%$ FCS, $2 \mathrm{mmol} / \mathrm{L}$ L-glutamine, $50 \mathrm{IU} / \mathrm{mL}$ penicillin, and $50 \mu \mathrm{g} / \mathrm{mL}$ streptomycin in a humidified atmosphere containing $5 \% \mathrm{CO}_{2}, 16 \% \mathrm{O}_{2}$, and $79 \% \mathrm{~N}_{2}$ at $37^{\circ} \mathrm{C}$. The cell line, BT-549, was maintained in RPMI1640 medium. When indicated, the cells were incubated under hypoxic conditions in a Ruskinn Sci-Tive-N hypoxia workstation under $5 \%$ or $1 \% \mathrm{O}_{2}$, $5 \% \mathrm{CO}_{2}$ balanced with $\mathrm{N}_{2}$ for 6 hours.

\section{Plasmids and site-directed mutagenesis}

pClneo-Myc-CIN85 D111G was a gift from Yutaka Hata (Addgene plasmid catalog no. 47935), the D114G mutation was eliminated by site-directed mutagenesis (QuikChange Mutagenesis Kit, Promega). The constructs for the bimolecular fluorescence complementation assay (BiFC) were generated by PCR, the respective PCR products were subcloned into the BamHI and $X b a \mathrm{I}$ sites (for PHD2) or EcoRI and XbaI sites (for CIN85) of pcDNA3-YN [nonfluorescent N-terminus of yellow-fluorescent protein (YFP)] or pcDNA3-YC (nonfluorescent C-terminus of YFP) plasmids, respectively (14). The constructs encoding CIN85 deletion variants and the ones allowing the expression of the recombinant GST-SH3A, GST-SH3B, GST-SH3C, GST-Pro, GSTSer, and GST-CC fusion proteins were described previously (15). The expression vectors for PHD2, PHD1, and PHD3 were described previously (16) and further details are included as Supplementary Information.

\section{Protein preparation and Western blotting}

The cells were lysed in lysis buffer $[50 \mathrm{mmol} / \mathrm{L}$ Gris-HCl, $\mathrm{pH}$ $7.5,150 \mathrm{mmol} / \mathrm{L} \mathrm{NaCl}, 1 \%$ Triton X-100, $1 \mathrm{mmol} / \mathrm{L} \mathrm{o}$-vanadate, $50 \mathrm{mmol} / \mathrm{L} \mathrm{NaF}, 2 \mathrm{mmol} / \mathrm{L} \rightarrow$ DTA, $1 \mathrm{mmol} / \mathrm{L}$ PMSF, complete protease inhibitor cocktail tablet (Roche)], kept on ice for 10 minutes and centrifuged at $14,000 \times g$ for 20 minutes at $4^{\circ} \mathrm{C}$. For EGF stimulation, cells were cultured in starvation medium (DMEM containing $0.1 \%$ FBS) for 24 hours, then treated with EGF $(100 \mathrm{ng} / \mathrm{mL})$, and lysed as described above. The levels of HIF1 $\alpha$, HIF2 $\alpha$, PHD1, PHD2, PHD3, Myc-CIN85, V5-PHD1, V5-PHD2, V5-PHD3, Flag-CIN85, CIN85, phospho-AKT, and phospho-ERK1/2 were detected by Western blotting from whole-cell extracts. Proteins $(20-100 \mu \mathrm{g}$ per sample) were separated by electrophoresis on $7.5 \%-12 \%$ polyacrylamide gels and transferred to nitrocellulose membranes. The membranes were incubated with the following antibodies: HIF1 $\alpha$ (catalog no. 610959, BD Biosciences), HIF2 $\alpha$ (catalog no. NB100-122, Novus Biologicals), PHD1 (NB100-310, Novus Biologicals), PHD2 (catalog no. 3293, Cell Signaling Technology), PHD3 (NB100139, Novus Biologicals), Myc-Tag (catalog no. 2278, Cell Signaling Technology), V5-Tag (catalog no. R96025, Thermo Fisher Scientific), Flag M2 (catalog no. F1804, Sigma-Aldrich,), CIN85 (catalog no. 12304, Cell Signaling Technology), phospho-AKT (pSer473) (catalog no. 9271, Cell Signaling Technology), AKT (catalog no. 9272, Cell Signaling Technology), phospho-ERK1/2 (pThr202/ pTyr204) (catalog no. 9101, Cell Signaling Technology), ERK1/2 (catalog no. 9107, Cell Signaling Technology), $\alpha$-tubulin (B-5-1-2) (catalog no. T5168, Sigma-Aldrich), CD2AP (catalog no. 25272, Santa Cruz Biotechnology), and $\beta$-actin (A5316, Sigma-Aldrich) overnight at $4^{\circ} \mathrm{C}$. Appropriate secondary antibodies (peroxidase-conjugated IgG, Bio-Rad) were used. The ECL Kit (GE Healthcare) was used for signal detection. Blots were quantified by densitometry with the Image Quant TL Program (GE Healthcare); densitometry data were normalized to $\alpha$-tubulin or $\beta$-actin.

\section{Immunoprecipitation}

For the coimmunoprecipitation of CIN85 and PHDs, HEK-293 cells were transiently transfected with expression plasmids 
encoding Myc-tagged CIN85 and V5-tagged PHDs (PHD1, PHD2, and PHD3) or Flag-tagged CIN85 lacking either SH3A, SH3B, SH3C, or all three SH3 domains, and V5-tagged PHD2. Immunoprecipitations were carried out as described previously (3). Cells were harvested 48 hours posttransfection, washed twice with ice-cold PBS, and lysed as described above. Aliquots of cleared HEK-293 cell lysates containing $1 \mathrm{mg}$ of total protein were mixed with protein G Sepharose beads (GE Healthcare) and Myc-tagged CIN85 was immunoprecipitated with the Myc-tag antibody; the Flag-tagged CIN85 variants were immunoprecipitated with the Flag $\mathrm{M} 2$ antibody at $4^{\circ} \mathrm{C}$ overnight. For the coimmunoprecipitation of CIN85 and PHD2 at the endogenous level, CIN85 was immunoprecipitated from the lysates of MDA-MB-231, Hs 578T, and BT-549 cells with the SH3A-CIN85 mAb (17) or CD2AP (catalog no. 25272, Santa Cruz Biotechnology). The next day the beads were washed 5 times with lysis buffer, the immune complexes were then resolved by SDS-PAGE, and analyzed with antibodies against the Myc and V5 epitope, or PHD2.

\section{In vitro hydroxylation assay}

The catalysts [PHD2 wild-type (wt), $452-98$ PHD2, and PR mut 2] in pcDNA vectors containing a T7 promoter were in vitro transcribed and translated (IVTTed) in rabbit reticulocyte lysate by using the TnT Quick Coupled Transcription/Translation System (Promega) in the presence of $\left[{ }^{35} \mathrm{~S}\right]$ Met. An aliquot of the IVTTed catalysts was analyzed on a $10 \%$ SDS-page gel to ensure equal expression levels. The rest of the IVTTed catalysts $(45 \mu \mathrm{L})$ was used to in vitro hydroxylate $\left[{ }^{3} \mathrm{H}\right]$ Pro-labelled HIF1 $\alpha$-ODDD in the presence of cofactors $\mathrm{Fe}^{2+}(5 \mu \mathrm{mol} / \mathrm{L}), 2$-oxoglutarate $(320 \mu \mathrm{mol} / \mathrm{L})$, and ascorbate $(2 \mathrm{mmol} / \mathrm{L})$ at $37^{\circ} \mathrm{C}$ for 30 minutes. The generation of $\left[{ }^{3} \mathrm{H}\right]$ 4-hydroxyproline was determined by a radiochemical assay (for details see ref. 18).

\section{Xenograft mouse model}

A total of $5 \times 10^{5}$ cells of CRISPR/Cas9-generated MDA-MB231 cells expressing edited EGLN1 (PHD2) (E10 and E12) together with scrambled (S) control cells were injected in the thoracic and inguinal mammary fat pad of 4-week-old female athymic nude mice (Envigo). The mice were housed in individually ventilated cages with water and food ad libitum for up to 5 weeks. The animals were euthanized by $\mathrm{CO}_{2}$ inhalation and additional cervical dislocation, and the tumors were collected. All animals were housed in the laboratory Animal Center of the University of Oulu (Oulu, Finland) in specific pathogen-free facilities on a 12-hour light/dark cycle, at a constant temperature of $22^{\circ} \mathrm{C}$. The protocol for animal use and experiments was approved by the National Animal Experimental Board of Finland, as well as the Animal Welfare Body of the Laboratory Animal Center and conducted according to the EU directive 2010/63/EU. The volume of the tumors formed was calculated as described previously (19).

\section{Statistical analysis}

The results are presented as mean values \pm SD of at least three independent experiments. Statistical analyses were performed using Student two-tailed $t$ test. Differences of $P \leq 0.05$ were considered statistically significant.

\section{Supplementary material summary}

Supplementary material consists of one table listing the sequences of the oligonucleotides used in the study followed by six supplementary figures containing the results of the GST-pull downs between CIN85 and PHD2 fragments (Supplementary Figs. S1 and S2), generation of E10 and E12 MDA-MB-231 cells lacking the CIN85-PHD2 interaction part of PHD2 (Supplementary Fig. S3), Western blot data and qRT-PCR from CRISPR/Cas9-edited EGLN1 cells (Supplementary Figs. S5 and S6), proliferation and wound-healing assay data from CRISPR/Cas9-edited EGLN1 cells in the presence of FG-4592, overexpressed HIF1 $\alpha$, and PHD2 (Supplementary Fig. S7), and analyses of the off-target effects in the CRISPR/Cas9-edited EGLN1 cells (Supplementary Fig. S8). Plasmids and site-directed mutagenesis, GST-pull down, fluorescence microscopy, CRISPR/ Cas9-mediated EGLN1 gene editing, lentivirus-mediated expression of single guide RNAs (sgRNA) and Cas9, genomic DNA extraction and genotyping, RNA extraction and qRT-PCR, scanning electron microscopy, monolayer colony formation assay, and live cell imaging assays surface plasmon resonance (SPR) are listed in Supplementary Materials.

\section{Results}

\section{CIN85 and PHD2 undergo a direct interaction}

We hypothesized that CIN85 contributes to HIF1 $\alpha$ stabilization by interfering with the function of PHDs via a direct interaction and explored this in more detail. Because PHD2 is the major variant regulating HIF1 $\alpha$ levels (20), we first performed coimmunoprecipitation studies in the three triplenegative breast cancer cell lines MDA-MB-231, BT-549, and Hs 578T. The results show that endogenous CIN85 and PHD2 interact in all three cell lines (Fig. 1A). Next, we investigated whether CIN85 is also able to interact with PHD1 and PHD3, which in contrast to PHD2 do not possess an N-terminal MYND (myeloid, Nervy, and DEAF-1) zinc-finger domain. To determine this, we performed coimmunoprecipitation studies in HEK-293 cells transiently overexpressing V5-tagged PHD1, PHD2, and PHD3 together with Myc-tagged CIN85. The coimmunoprecipitation assays revealed that only PHD2 binds to CIN85; no interaction of CIN85 with PHD1 or PHD3 could be detected (Fig. 1B). Thus, these data indicate that CIN85 may interfere with HIF $\alpha$ degradation only via PHD2 and not via PHD1 or PHD3.

We next localized the PHD2-CIN85 complex in living cells by performing a BiFC. The BiFC assay is based on the formation of a fluorescent complex when two proteins, fused to the noninteracting and nonfluorescent parts of, in our case, the N-terminal and C-terminal halves of YFP, interact with each other (14). Upon that interaction, the YFP fluorescent complex is reconstituted and can be visualized. For this purpose, coding sequences of CIN85 and PHD2 were subcloned into pcDNA3-CMV-YN and pcDNA3-CMV-YC constructs, allowing the expression of PHD2 and CIN85 fused to the N-terminal or the C-terminal nonfluorescent parts of YFP, respectively. A description of the constructs used in the BiFC assay is schematically presented in Fig. 1C. BT549 cells were transfected with the CMV-CIN85-YN and CMVPHD2-YC constructs. Transfection with a combination of unfused CMV-YN + CMV-YC constructs served as a negative control, whereas transfection with a construct, encoding full length YFP served as a positive control. A punctated PHD2-CIN85 BiFC signal indicating the interaction of PHD2 with CIN85 could be mainly visualized throughout the cytoplasm and to a lesser extent in the nucleus of the transfected cells (Fig. 1D). Thus, these data indicate 
A

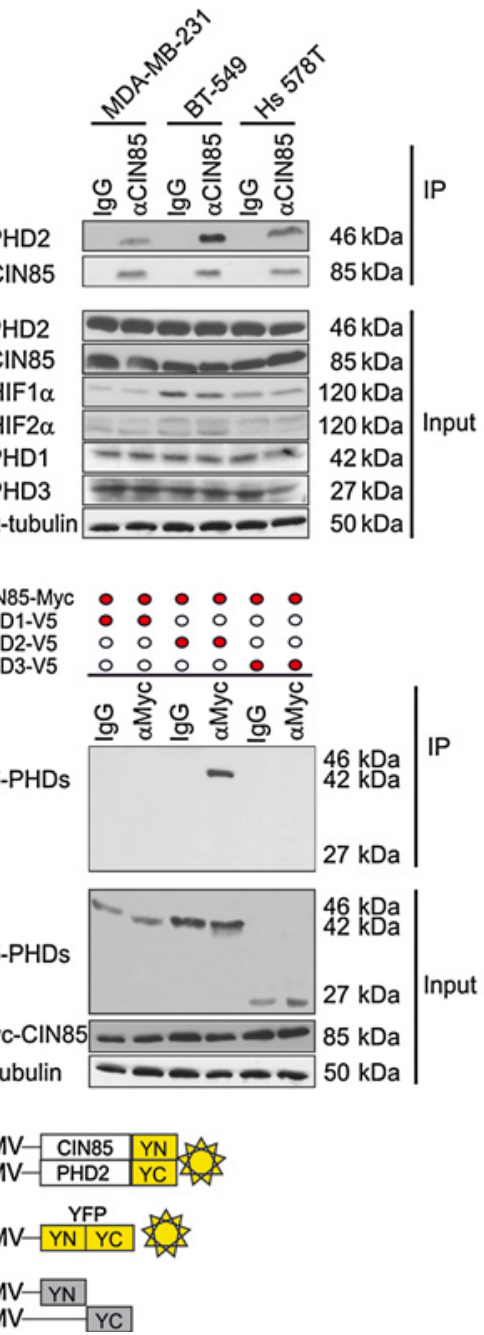

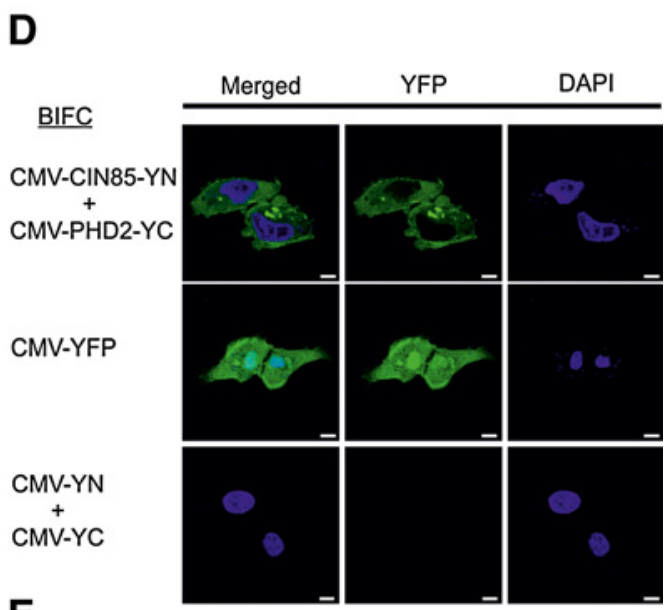

$\mathbf{E}$
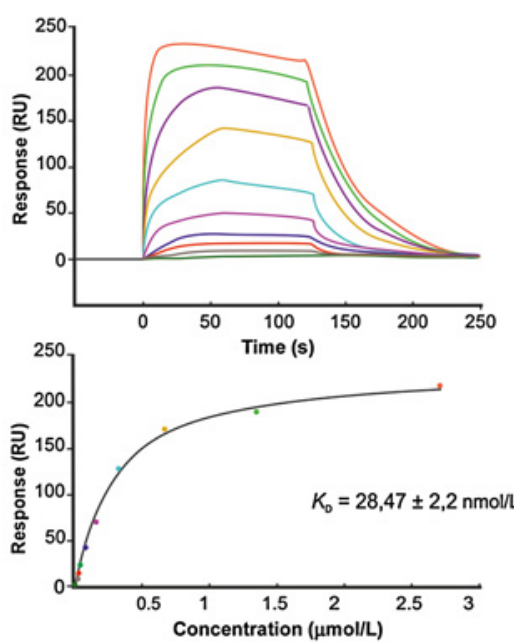

Figure 1.

CIN85 interacts with PHD2. A, Endogenous PHD2 was immunoprecipitated (IP) with CIN85 antibody from MDA-MB-231, BT-549, and Hs 578T cells and probed for PHD2 and CIN85. Blots from the input were probed with PHD1, -2, -3, CIN85, HIFl $\alpha$, and HIF2 $\alpha$ antibody. B, Western blot analysis of immunoprecipitates and inputs from HEK-293 cells expressing V5-tagged PHD1, PHD2, or PHD3 and Myc-tagged CIN85. Blots from immunoprecipitates were probed with V5-tag antibody; the input was probed with V5-tag, Myc-tag, and $\alpha$-tubulin antibodies. C, Schematic presentation of BiFC assay constructs. The constructs CMV-CIN85YN and CMV-PHD2-YC allow expression of CIN85 and PHD2 as fusion proteins with the N-terminal or the C-terminal nonfluorescent parts of YFP (-YN and -YC) under the control of the CMV promoter, respectively. Note that CMV-YN and CMV-YC protein parts are nonfluorescent and noninteracting; however, interacting proteins such as CIN85 and PHD2 are able to reconstitute fluorescent YFP. D, Visualization of the BiFC signal by confocal microscopy in BT-549 cells expressing CMV-CIN85-YN + CMV-PHD2-YC. No signal was detected upon expression of CMV-YN + CMV-YC. Scale bar, $20 \mu \mathrm{m}$. E, The surface plasmon resonance sensorgram of CIN85 binding to immobilized PHD2. Binding was assessed upon injection of a concentration series of CIN85 over immobilized PHD2. The CIN85 concentrations were $0,10,21,42,84,168,337,675,1,350$, and $2,700 \mathrm{nmol} / \mathrm{L}$ (from bottom to top). F, The fitted curve for different concentrations of CIN85 binding to immobilized PHD2 using the "Affinity" model in the Biacore T200 evaluation software.

that PHD2 interacts with CIN85 predominantly in the cytoplasmic part of the cells.

To understand whether the interaction between PHD2 and CIN85 is direct, we performed SPR experiments. To this end, PHD2 was covalently immobilized to a SPR sensor chip, and a concentration series of recombinant CIN85 was injected over PHD2 and binding was assessed by SPR detection. The measurements revealed that fullength CIN85 bound to immobilized PHD2 with an apparent $K_{\mathrm{d}}$ of $28.47 \pm 2.2 \mathrm{nmol} / \mathrm{L}$ (Fig. 1E and F). Together, the data show that PHD2 and CIN85 interact in a direct manner.
The three N-terminal SH3 domains of CIN85 bind to the $\mathrm{N}$-terminus of PHD2

Next, we investigated which parts of each protein are involved in the interaction. CIN85 is a multi-domain adaptor protein consisting of three SH3 domains (A, B, and C), a proline-rich domain (Pro), and a serine-rich sequence (Ser), followed by a coiled-coil (CC) domain. PHD2 harbors a flexible N-terminus with the MYND zinc-finger domain and a catalytic domain on the C-terminus (Fig. 2A).

First, we performed GST-pull down experiments with recombinant GST-SH3A, GST-SH3B, GST-SH3C, GST-Pro, GST-Ser, and 
Kozlova et al.
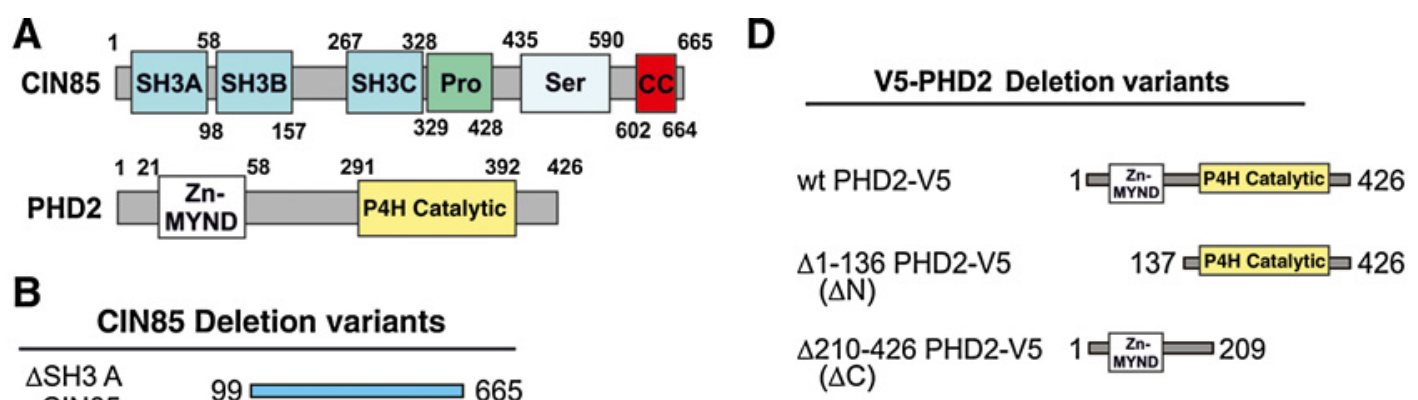

B

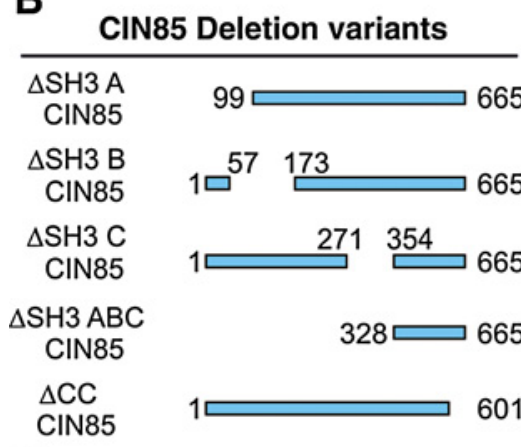

$(\Delta \mathrm{C})$

E

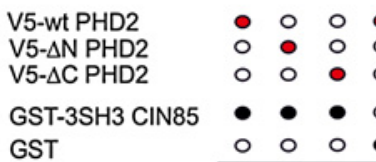

GST

V5-PHD2

Variants

C

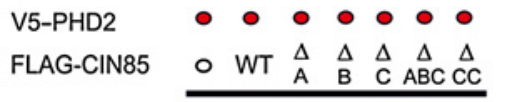

V5-PHD2

CD2AP

CIN85
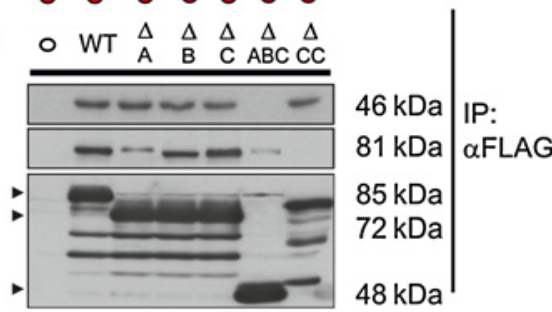

Variants
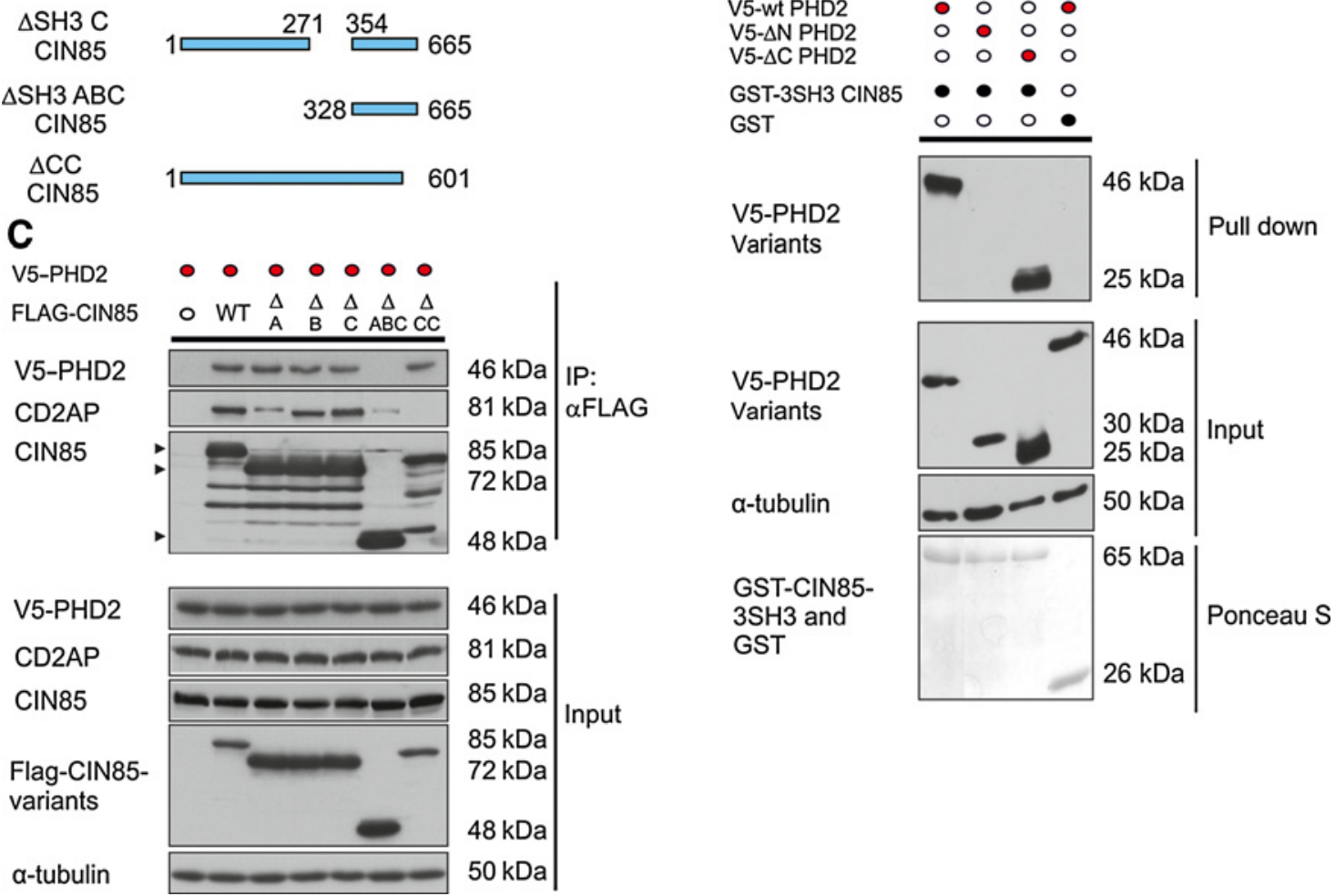

V5-PHD2

Variants

a-tubulin

GST-CIN85-

$3 \mathrm{SH} 3$ and

GST

$50 \mathrm{kDa}$

Figure 2.

CIN85 interacts with the N-terminus of PHD2 via 3 SH3 domains. A, Schematic presentation of the CIN85 and PHD2 structure. CIN85 consists of three N-terminal $\mathrm{SH} 3$ domains ( $\mathrm{A}, \mathrm{B}$, and $\mathrm{C}$ ), a proline-rich domain (Pro), and a serine-rich sequence (Ser), followed by a CC domain. The $\mathrm{N}$-terminus of PHD2 possesses a MYNDzinc-finger domain, while the catalytic domain of PHD2 is located in the C-terminus. B, Schematic presentation of FLAG-tagged CIN85 variants with a deletion of either the SH3 A, SH3 B, SH3 C, or all three SH3 domains, as well as the CC domain used in the immunoprecipitation (IP) studies. C, Western blot analysis of immunoprecipitates and inputs from HEK-293 cells expressing V5-tagged PHD2 variants and FLAG-tagged CIN85 variants. Blots from immunoprecipitates were probed with V5-tag antibody, CD2AP, and CIN85 antibody; extracts were probed with V5-tag, CD2AP, CIN85, FLAG-tag, and $\alpha$-tubulin antibodies. Arrows, CIN85 bands. D, Schematic presentation of the V5-tagged PHD2 variants used in GST pull-down studies: wt PHD2, PHD2 lacking amino acids 1-136 from the N-terminus $(\Delta \mathrm{N})$, and PHD2 variant lacking amino acids 210-426 from the C-terminus $(\Delta \mathrm{C})$. E, Western blot analysis of pull downs using recombinant GST or GST fusion $3 S H 3$ CIN85 protein in extracts from HEK-293 cells expressing V5-tagged PHD2 variants (wt, $\Delta \mathrm{N}$, or $\Delta \mathrm{C}$ ). Blots from pull downs were probed with V5-tag antibody; inputs were probed with V5-tag and $\alpha$-tubulin antibodies. Ponceau S stain of blots from pull downs was used as a loading control.

GST-CC fusion proteins and lysates of HEK-293 cells transiently expressing V5-PHD2 (Supplementary Fig. S1A). As a result, we were able to identify that the SH3 domains from CIN85 are responsible for the interaction with PHD2 (Supplementary Fig. S1B), while no binding was observed between PHD2 and other domains of CIN85.

We next tested whether the CIN85-PHD2 interaction will be affected by the lack of a particular SH3 domain in CIN85. To do that, coimmunoprecipitations with FLAG-tagged CIN85 and mutants lacking either SH3A, SH3B, SH3C, or all three $\mathrm{SH} 3$ domains, as well as the CC domain together with V5-tagged PHD2 were performed in HEK-293 cells (Fig. 2B). We found that the CIN85-PHD2 interaction was present with full-length CIN85 or when CIN85 lacked either the SH3A, SH3B, SH3C, or CC domain; however, absence of all three $\mathrm{SH} 3$ domains abolished the interaction (Fig. 2C).

The overall domain organization of CIN85 displays high sequence and structural similarities to CD2-associated protein 
(CD2AP). Although significant differences between CIN85 and CD2AP, such as the presence of actin-binding sites in CD2AP exist, there may be also similar binding properties that could result in functional redundancies between the two family members $(21,22)$. Thus, to examine whether CD2AP could be part of the CIN85 and PHD2 complex, we probed the coimmunoprecipitation experiments for presence of CD2AP. The data show that CD2AP appeared in the same complex with PHD2. However, binding between PHD2 and CIN85 but not binding between $\mathrm{CD} 2 \mathrm{AP}$ and CIN85 was lost in the coimmunoprecipitations with CIN85 constructs lacking all three SH3 domains. In contrast, we lost binding of CD2AP but not binding of PHD2 upon usage of the CIN85 construct lacking only the CC domain (Fig. 2C). Together, these data indicate that the interaction of PHD2 with CIN85 is mediated via the SH3 domains of CIN85 and not of those from CD2AP, and that binding between CIN85 and CD2AP involves the CC domain.

Next, we investigated which part of PHD2 participates in the binding to CIN85, and used, in addition to V5-tagged wt PHD2, two constructs that allow the expression of either a V5-tagged $\mathrm{N}$ terminal part of PHD2 (V5- $\Delta \mathrm{C}$ PHD2) or a V5-tagged C-terminal part (V5- $\Delta \mathrm{N}$ PHD2; Fig. 2D). In addition, because all three SH3 domains from CIN85 participate in the complex formation, we used a recombinant GST-3SH3-CIN85 protein in pull-down studies as bait for the PHD2 deletion variants (Fig. 2E). The results of the pull-down assays show that GST-3SH3-CIN85 was able to interact with wt PHD2 and the PHD2 variant lacking the catalytic C-terminus (V5- $\Delta$ C PHD2). In contrast, no interaction was observed with a PHD2 lacking the first 136 amino acids of the $\mathrm{N}$-terminus (V5- $\Delta \mathrm{N}$ PHD2; Fig. 2E). Together, these data show that the interaction between PHD2 and CIN85 occurs between the three SH3 domains of CIN85 and the amino acids 1-136 within the N-terminus of PHD2.

CIN85 binds a PR-rich sequence in the N-terminus of PHD2

To further delineate the CIN85-binding PHD2 region, we generated a series of PHD2 coding constructs lacking various amino acids from the N-terminus of PHD2 (Fig. 3A) and GSTfusion proteins harboring peptides of different length from the PHD2 N-terminus (Supplementary Fig. S2A and S2C). The latter were used for pull downs with lysates of HEK-293 cells transiently expressing full length Myc-CIN85 (Supplementary Fig. S2A and S2C). As a result, GST-1-40-PHD2 and GST-99-136-PHD2 were not able to pull down CIN85, indicating that amino acids 1-40 and $99-136$ are not critical for the PHD2-CIN85 interaction (Supplementary Fig. S2B). Other GST-PHD2 fragments used in the study were able to bind CIN85, although the binding was most evident when the amino acids 59-98 were present within the PHD2 fragment. Further pull down studies with shorter GSTPHD2 variants (Supplementary Fig. S2C) revealed that GST-8198-PHD2 showed the strongest binding to CIN85, while other GST proteins were not able to pull down CIN85 to a similar level (Supplementary Fig. S2D). Altogether the results of the pull down studies indicated that the PHD2 region between amino acids 5898 is necessary for the binding to CIN85, whereas amino acids 8198 have the strongest impact on the interaction with CIN85. Indeed, we observed similar results when using recombinant GST-3SH3-CIN85 as bait in the lysates of HEK-293 cells transiently expressing V5-tagged PHD2 variants lacking amino acids from the N-terminus (Fig. 3A and B). While the PHD2 variants $\Delta 1-26, \Delta 10-58, \Delta 99-136$, and $\Delta 210-426$ were able to bind
CIN85 like wt PHD2, the PHD2 variant lacking amino acids 52-98 showed an almost complete loss of binding like the PHD2 variant lacking the whole N-terminus (Fig. 3A and B; Supplementary Fig. S2E).

The SH3 domains of CIN85 are reported to recognize atypical PR sequences (23) with each of the domains tolerating different amino acid adjacent to the arginine residue. To further unravel the nature of the CIN85-PHD2 binding, we aimed to identify the proline and/or arginine residues within the region between amino acids 59-100 of PHD2 that are critical for the binding to CIN85. To verify their involvement in the binding, we created several V5tagged PHD2 constructs where the respective proline or/and arginine residues were mutated to alanines (referred to $6 \mathrm{P}$ mut, PR mut1, and PR mut2; Fig. 3A). Pulldown studies with these mutants and GST-3SH3-CIN85 revealed that these point-mutated PHD2 variants lost almost all their binding to CIN85 when compared with wt PHD2 (Fig. 3B). With the 6P mut variant, where prolines $77,78,80,84,85$, and 86 were substituted with alanines, binding was decreased by about $60 \%$. When PR mut 1 (P86, R87, R91, P93, and R94 to alanine) was employed binding was reduced by about $80 \%$. Finally, the employment of PR mut 2 (P86, P87, R91, P93, P94, R99, and R100 to alanine) reduced the binding between PHD2 and CIN85 by about 90\% (Supplementary Fig. S2E). Taken together, the results indicate that the proline and arginine residues located within the area of amino acids 77100 in the N-terminus of PHD2 are critical for the interaction with the SH3 domains of CIN85.

PHD2 variants lacking CIN85-binding sequence show higher catalytic activity

Because our previous findings indicated that CIN85 stabilizes HIF $1 \alpha$ levels via interference with PHD-dependent HIF $1 \alpha$ protein degradation, we investigated whether the ability of PHD2 to interact with CIN85 has an impact on the catalytic activity of PHD2. The results of an in vitro HIF1 $\alpha$ peptide hydroxylation assay (see Materials and Methods) with recombinant PHD2 as catalyst revealed that addition of recombinant full-length CIN85 reduces the catalytic activity of PHD 2 by about $70 \%$. In contrast, a CIN85 variant lacking the three SH3 domains involved in the interaction with PHD2 did no longer reduce the PHD2 catalytic activity. In addition, when we used the PHD2 variants with no or the weakest binding to CIN85 ( $\triangle 52-98$ PHD2; and PR mut2 PHD2, respectively) in the HIF1 $\alpha$ peptide hydroxylation assay, we observed that both PHD2 variants showed a higher activity (about $15 \%$ and $20 \%$, respectively) when compared with wt PHD2. Furthermore, the activity of the PHD2 mutants was not affected by the presence of CIN85 (Fig. 3C and D). In line with the results from the hydroxylation assay, overexpression of $\Delta 52-98$ and PR mut2 PHD2 increased the levels of hydroxy-HIF in MG123-treated cells (Fig. 3E). Furthermore, overexpression of $\Delta 52-98$ and PR mut2 PHD2 reduced HIF1 $\alpha$ levels under both normoxia and hypoxia, compared with wt PHD2 (Fig. 3F and G). Together, these results suggest that lack of CIN85 binding promotes PHD2 activity, and subsequently lowers HIF $\alpha$ levels.

\section{Generation of MDA-MB-231 cells lacking the CIN85-PHD2 interaction}

Next, we investigated the cellular impact of the CIN85-PHD2 interaction. To address this, we used the CRISPR/Cas9 approach in triple-negative MDA-MB-231 breast cancer cells to edit the EGLN1 (PHD2) gene in a manner that it encodes a PHD2 lacking 
Kozlova et al.

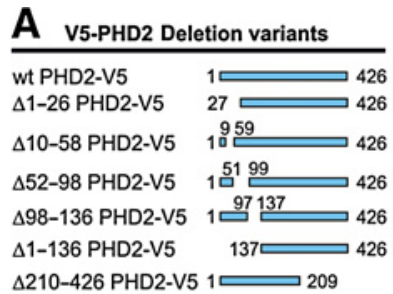

$\Delta 210-426$ PHD2-V5 $1{ }^{2} 209$
V5-PHD2 Point-mutated variants

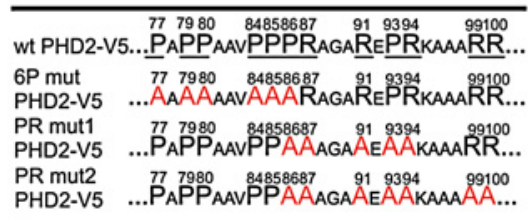

\section{B}

V5-PHD2

GST-3SH3

GST-3SH3
CIN85

V5-PHD2
Variants
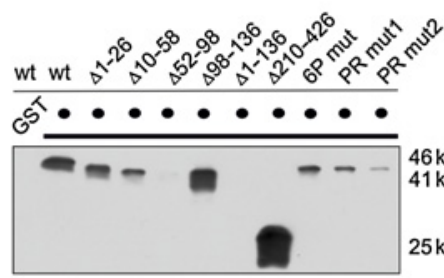

$46 \mathrm{kDa}$
$41 \mathrm{kDa}$

a

V5-PHD2
Variants

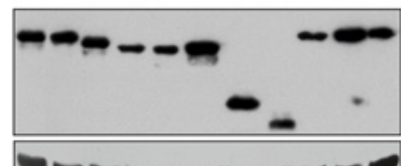

a-tubulin

Deverene

GST-CIN85-

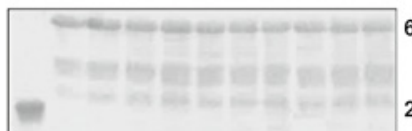

C

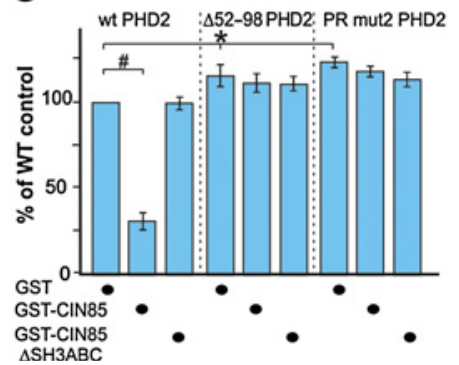

D

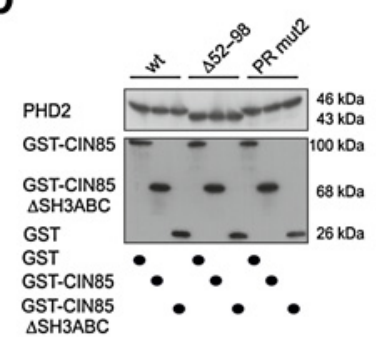

E

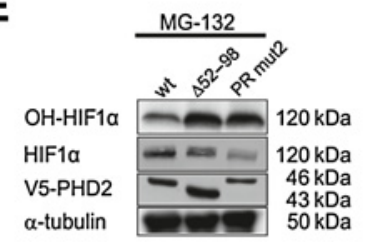

F

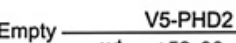

Empty $\begin{gathered}\text { V5-PHD2 } \\ \text { vector } \text { wt } \triangle 52-98 \text { PR mut2 }\end{gathered}$

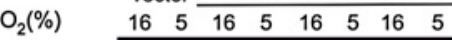

HIF1a $=120 \mathrm{kDa}$

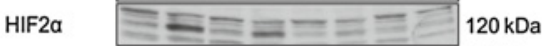

V5-PHD2 $-20-16 \mathrm{kDa}$

$\beta$-actin $42 \mathrm{kDa}$

G

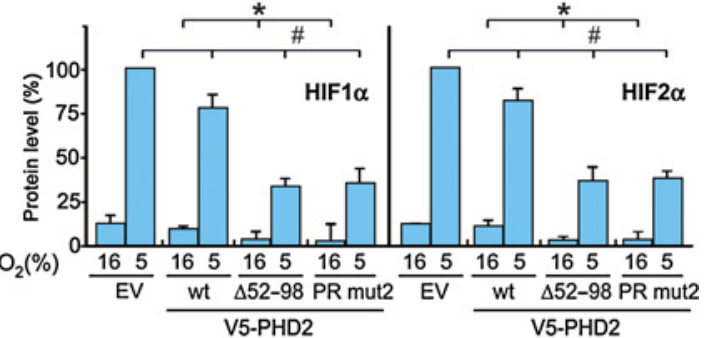

Figure 3.

The PHD2 variants lacking the CIN85-binding pattern are of higher catalytic activity. A, Schematic presentation of the V5-tagged PHD2 deletion variants and V5tagged point-mutated PHD2 variants used in the pull downs: wt PHD2, 1-26 ( $\Delta 1-26$ PHD2), 10-58 ( $\Delta 10-58$ PHD2), 52-98 ( $\Delta 52-98$ PHD2), $99-136$ ( $\Delta 99-136$ PHD2), 1-136 (41-136 PHD2), 209-426 (4210-426 PHD2), PHD2 P77A, P78A, P80A, P84A, P85A, P86A (6P), PHD2 P86A, R87A, R91A, P93A, R94A (PR mut1), and PHD2 P86A, P87A, R91A, P93A, P94A, R99A, R100A (PR mut2). B, Western blot analysis of pull downs using recombinant GST- 3SH3 CIN85 fusion proteins from HEK-293 cells expressing V5-tagged PHD2 variants. Blots from pull downs were probed with V5-tag antibody; the input was probed with V5-tag and $\alpha$-tubulin antibodies. Ponceau S stain of pull downs was used as a loading control. C, Results of the in vitro hydroxylation assay using wt PHD2, $\triangle 10-58$ PHD2, and PR mut2 PHD2 as catalysts and a [3H]proline-labeled HIF1 $\alpha$ peptide as a substrate in the presence of recombinant GST-CIN85 and GST-CIN85 4 SH3ABC. Recombinant GST was used as a control. The amount of 4-hydroxy [3H]proline formed was analyzed by a radiochemical assay (see Materials and Methods) and used as a read out of the PHD2 activity. Wt PHD2 + GST was set to $100 \%$. Data are mean \pm SD $(n=3) ;{ }^{*}$, significant difference between $\Delta 10-58$ PHD2, and PR mut2 versus wtPHD2; \#, significant difference between wt PHD2 and wt PHD2 + CIN85. D, Autoradiography of the $\left[^{35}\right.$ S]Met-labeled wt PHD2, $110-58$ PHD2, and PR mut2 PHD2 used in the hydroxylation assay. E, Hydroxy-HIF1 $\alpha$ levels in MG132-treated cells expressing wt PHD2, $\triangle 10-58$ PHD2, and PR mut2 PHD2. F, Western blot analysis of HEK-293 cells transiently expressing V5-tagged wt PHD2, $\Delta 10-58$ PHD2, and PR mut2 PHD2 cultured under normoxia (16\%) or hypoxia (5\% $\mathrm{O}_{2}$ ) for 6 hours. Extracts were probed with HIF1 $\alpha, \mathrm{HIF} 2 \alpha$, V5, and $\alpha$-tubulin antibodies. G, Quantification of HIF1 $\alpha$ levels in HEK-293 cells transiently expressing empty control vector (EV), V5-tagged wt PHD2, $\triangle 10-58$ PHD2, and PR mut2 PHD2 cultured under normoxia or hypoxia. HIF1 $\alpha$ and HIF2 $\alpha$ levels in empty control vectorexpressing cells cultured under hypoxia were set to $100 \%$. Data are mean $\pm \operatorname{SD}(n=4)$; ${ }^{*}$, significant difference between $\Delta 10-58$ PHD2, and PR mut2 PHD2expressing cells versus wt PHD2 (normoxia); \#, significant difference between HIF1 $\alpha$ levels in wt PHD2, $\Delta 10-58$ PHD2, and PR mut2 PHD2-expressing cells versus empty control vector-expressing cells (hypoxia). 
the amino acids required for binding to CIN85. By introducing two double-strand breaks in EGLN1 exon 1, this approach allowed deletion of 87 nucleotides (see Materials and Methods; Supplementary Fig. S3A).

The results of genotyping indicated the presence of various gene editing patterns after the introduction of Cas9 together with EGLN1-sgRNA-219 and EGLN1-sgRNA-292 into MDA-MB-231 cells (Supplementary Fig. S3B). However, the respective PCR products from two clones (E10 and E12) appeared as a single band of the correct size on native PAGE. Furthermore, we extracted total RNA from E10 and E12 cells and performed RTPCR using the same set of genotyping primers. The PCR products from both clones appeared to be of shorter size compared with the PCR product from S control cells (Supplementary Fig. S3C). Afterwards, the PCR products from the gDNA and cDNA of both clones were sequenced. As a result of sequencing, we observed only one type of template from both E10 and E12 MDA-MB-231 cells, which harbored a homozygous deletion of $87 \mathrm{bp}$ and encodes a PHD2 variant lacking amino acids 75-103 (Supplementary Fig. S3D). Next, by performing a Western blot analysis, we were able to confirm that the deletion of $87 \mathrm{bp}$ in exon 1 of EGLN1 allowed the expression of a smaller $\Delta 75-103$ PHD2 when compared with the wt PHD2 in control scrambled cells (S; Supplementary Fig. S3E).

Lack of amino acids 75-103 of PHD2 reduces HIF $\alpha$ levels

As a next step, we performed immunoprecipitations and GSTpull downs using CIN85 antibodies and GST-3SH3-CIN85, and verified that the PHD2-CIN85 interaction in the E10 and E12 MDA-MB-231 cells was lost (Fig. 4A and B).

Because our previous data showed that lack of the CIN85binding domain within PHD2 increases its activity and reduces HIF1 $\alpha$ (Fig. 3C-G), we now analyzed whether the lack of the CIN85-binding site in the E10 and E12 cells increased the levels of hydroxy-HIF1 $\alpha$. The data show that upon inhibition of the proteasomal degradation with MG132, increased levels of hydroxy-HIF1 $\alpha$ could be detected (Fig. 4C). Next, we tested whether the edited cells would display reduced protein levels of HIF $1 \alpha$ and HIF2 $\alpha$ under normoxia or hypoxia. Indeed, HIF $1 \alpha$ protein levels were reduced by about $50 \%-60 \%$ in E10 and E12 cells compared with control S cells under normoxia and by about $75 \%$ under hypoxia (Fig. 4D and E). In addition, HIF $2 \alpha$ protein levels were also downregulated in E10 and E12 cells. While there was a trend for decreased HIF2 $\alpha$ levels under normoxic conditions, both E10 and E12 cells showed about $60 \%$ lower HIF $2 \alpha$ induction under hypoxia (Fig. 4D and E).

In addition, we tested whether the reduction in HIF $\alpha$ levels has consequences on the expression of HIF target genes. Indeed, loss of the CIN85-PHD2 interaction in E10 and E12 cells reduced expression of LDHA, GLUT1, VEGF, and CITED2 mRNA under normoxia when compared with $S$ cells and severely reduced their hypoxia-dependent induction (Fig. 4F).

It is known, that hypoxia-triggered HIF $\alpha$ accumulation mainly arises from a PHD-mediated decrease in HIF degradation, rather than from an increase in mRNA expression $(20,24)$. To investigate whether the downregulation of HIF $1 \alpha$ and HIF $2 \alpha$ may result from a decrease in mRNA expression, we measured HIF $1 \alpha$ and HIF $2 \alpha$ mRNA expression by qRT-PCR. We did not detect any significant changes in the expression of HIF1 $\alpha$ mRNA between $S$ and E10 or E12 cells (Supplementary Fig. S4A). However, clone E12 expressed lower levels of HIF2 $\alpha$ mRNA compared with $S$ cells under normoxic conditions (Supplementary Fig. S4B).

To assess whether the absence of the amino acids 75-103 in the engineered cells would lead to a changed expression of the other PHD enzymes, we measured the protein levels of PHD1 and PHD3 by Western blot analysis. No differences were found in the expression of PHD1 and PHD3 in E10 or E12 cells (Supplementary Fig. S4C-S4E). In line with the results from the Western blot analyses, E10 and E12 cells did not show changes on the PHD1, PHD2, and PHD3 mRNA levels under normoxic conditions compared with control cells (Supplementary Fig. S4F-S4I). Because the expression levels of PHD1 and PHD3 were not changed in E10 and E12 cells (Supplementary Fig. S4FS4I), it is possible to conclude that that the observed downregulation in HIF $1 \alpha$ and HIF $2 \alpha$ levels is a result of the altered PHD2. Altogether, these data show that the lack of CIN85-PHD2 interaction contributes to a decrease in HIF $\alpha$ levels.

Lack of amino acids 75-103 in PHD2 mediates HIF $\alpha$ resistance to CIN85 overexpression and attenuates EGF-mediated HIF $\alpha$ induction

The abovementioned findings suggest that the HIF $\alpha$ levels in the E10 and E12 cells could be resistant to overexpression of CIN85 and that depletion of CIN85 could mimic the loss of the CIN85-PHD2 interaction. To test this, we overexpressed CIN85 in $\mathrm{S}, \mathrm{E} 10$, and E12 cells. The findings show that overexpression of CIN85 increased HIF1 $\alpha$ and HIF2 $\alpha$ levels only in the S cells but not in E10 and E12 cells (Fig. 5A and B). In addition, when we knocked-down CIN85 with shRNA we observed that loss of CIN85 decreased HIF $\alpha$-levels only in S cells, whereas the already lower HIF levels in E10 and E12 cells were not affected by the knockdown of CIN85 (Supplementary Fig. S5A and S5B).

Because CIN85 has a prime function in the activation of ERK1/2 and AKT in the EGF signaling pathway (11), we next investigated whether the loss of the interaction between PHD2 and CIN85 in the E10 and E12 cells affects ERK1/2 and AKT activation and induction of HIF $1 \alpha$ and HIF $2 \alpha$ in response to EGF. The results show that neither basal nor EGF-dependent activation of both ERK1/2 and AKT was altered in E10 and E12 cells when compared with $S$ cells. In addition, EGF mediated an increase in HIF1 $\alpha$ levels in the S cells of about 5-fold under normoxia. EGF was additive under hypoxia and enhanced the hypoxia-dependent induction by about 2 -fold. While EGF also mediated about 5-fold induction of HIF $2 \alpha$ in the S cells under normoxia, no additive effect was seen under hypoxia. Although EGF also caused an induction of both HIF1 $\alpha$ and HIF $2 \alpha$ in E10 and E12 cells under normoxia; this induction was less robust than in the $\mathrm{S}$ cells (Fig. 5C and D). Together, these data suggest that the loss of the PHD2-CIN85 interaction is rather selective for HIF $\alpha$ regulation and does not affect basal and EGF-dependent ERK1/2 and AKT regulation.

Lack of amino acids 75-103 in PHD2 alters cell morphology and contributes to less malignant cell properties

MDA-MB-231 is a triple-negative breast cancer cell line known to have an aggressive metastatic phenotype. Therefore, we further investigated whether the expression of $\Delta 75-103$ PHD2, and thus the lack of CIN85-PHD2 interaction, contributed to any changes in morphology and malignancy of these cells. To visualize the cell shape and surface composition, we imaged S, E10, and E12 cells by scanning electron microscopy (Supplementary Fig. S6A). Cells 

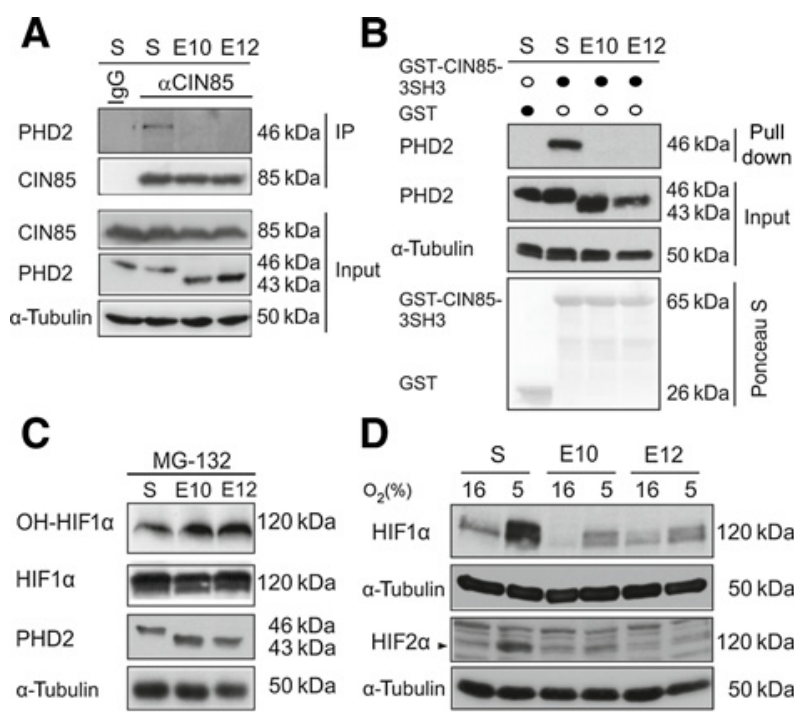

D
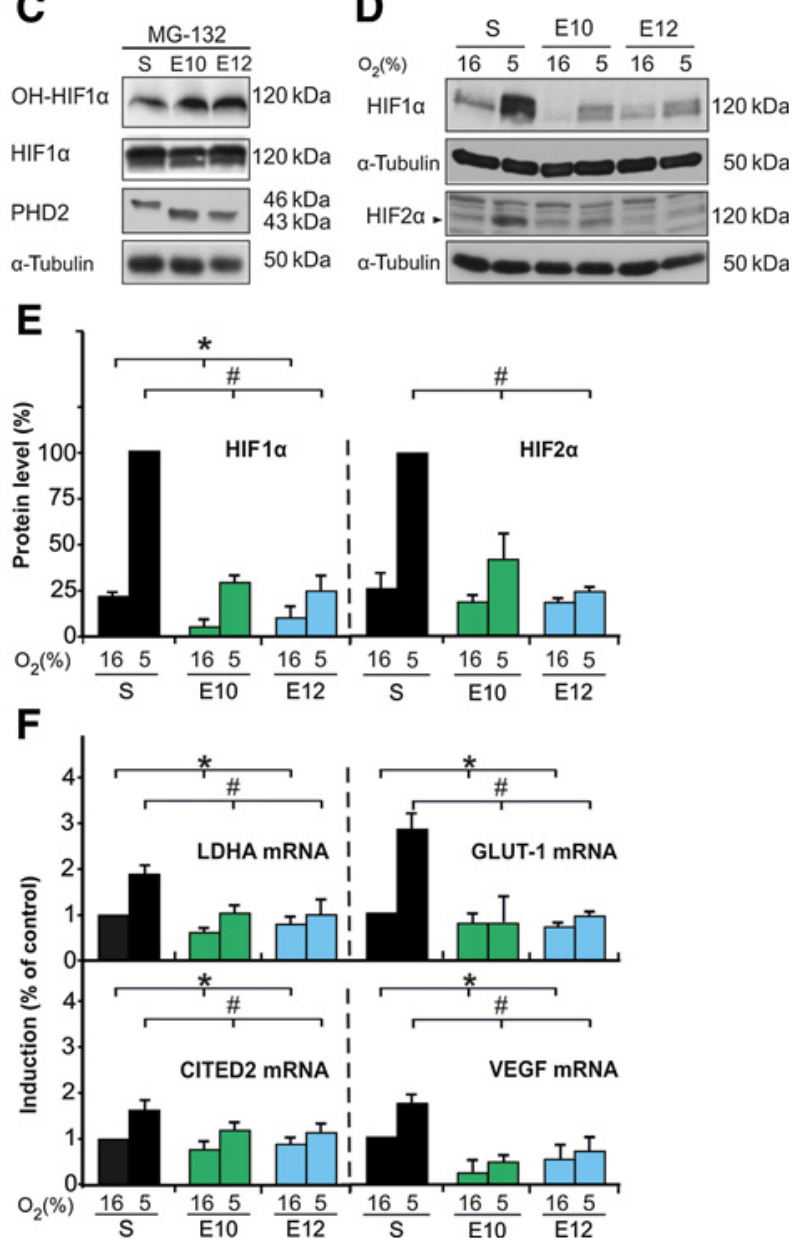

Figure 4.

Lack of amino acids 75-103 within PHD2 reduces HIF $\alpha$ levels in MDA-MB-231 cells. A, Immunoprecipitations (IP) were performed with the CIN85 antibody from control S, E10, and E12 cells and probed for PHD2 and CIN85. Blots from the input were probed with CIN85, PHD2, and $\alpha$-tubulin antibody. B, Western blot analysis of pull downs using recombinant GST and GST-fusion 3SH3 CIN85 proteins in MDA-MB-231 control S, E10, and E12 cells. Blots from pull downs were probed with PHD2 antibody; blots from the input were probed with PHD2 and $\alpha$-tubulin antibodies. Ponceau S stain was used as a loading control. C, Hydroxy-HIF1 $\alpha$ levels in MG132-treated control S, E10, and E12 cells. Extracts were probed with $\mathrm{OH}-\mathrm{HIF} 1 \alpha, \mathrm{PHD} 2$, and $\alpha$-tubulin antibodies. D, HIF1 $\alpha$ and HIF2 $\alpha$ levels in control S, E10, and E12 cells cultured under normoxia (16\%) or hypoxia $\left(5 \% \mathrm{O}_{2}\right)$ for 6 hours. Blots were probed with HIF1 $\alpha$, HIF2 $\alpha$, and $\alpha$-tubulin antibodies. E, Quantification of HIF1 $\alpha$ and HIF2 $\alpha$ levels in control S, E10, and E12 cells, cultured under normoxia or hypoxia. HIF1 $\alpha$ and HIF2 $\alpha$ levels in S cells cultured under hypoxia were set to 100\%. Data are mean \pm SD $(n=4)$; ${ }^{*}$, significant difference between E10 and E12 cells versus control (normoxia); \#, significant difference between $\mathrm{E} 10$ and $\mathrm{E} 12$ cells versus expressing $\Delta 75$-103 PHD2 were elongated with fewer protrusions on the leading edge of the cells. In addition, we investigated whether the absence of amino acids 75-103 had an impact on the intracellular localization of PHD2. Immunofluorescence revealed that $\Delta 75-103$ PHD2 did not change the cellular localization. The distribution was mainly cytoplasmic, which was similar to the wt PHD2 in S control cells. Visualization of $\alpha$-tubulin in S, E10, and E12 further verified the altered shape and morphology of E10 and E12 cells, in line with the results of the electron microscopy (Supplementary Fig. S6B). After observing the changes in cellular shape in E10 and E12 cells, we assessed cellular proliferation, the ability to form colonies, the motility of S, E10, and E12 cells, and tumor forming potential by injecting these cells into nude mice.

When performing live-cell proliferation analyses using the IncuCyte ZOOM System, we found that proliferation of the two clones E10 and E12, expressing $475-103$ PHD2 was slower than that of MDA-MB-231 S control cells (Fig. 6A). While it took 72 hours for the control S cells to reach $100 \%$ confluence, E10 cells were able to display the same confluence level only after 108 hours, whereas E12 cells showed only $80 \%$ confluence at the end of a 117 hour experiment (Fig. 6A).

In addition, we evaluated cellular motility in a low-serum wound (scratch) assay. Confluent MDA-MB-231 control S, E10, and E12 cells were wounded and wound closure was monitored for 72 hours. In line with the data from proliferation assays, both of the clones expressing $\Delta 75-103$ PHD2 were almost $50 \%$ less motile than the control cells during wound closure. While the control $S$ cells were able to close the scratch area in about 24-30 hours, it took E10 and E12 cells about 12-18 hours and 26-32 hours longer, respectively (Fig. 6B and $\mathrm{C}$ ).

The ability to grow and form colonies from single cells is a very important malignant property of cancer cells. Therefore, we performed colony formation assays by plating cells at very low density and allowing them to grow for 10 days. Colony formation was drastically impaired in E10 and E12 cells (Fig. 6D and E). The number and size of colonies formed by control S cells was higher than in E10 and E12 cells. In addition, the colonies of control cells were dense and displayed an overlapping growth pattern, while E10 and E12 cells were disseminated within the colony (Fig. 6D and E). Although the decrease in colony formation with the E10 and E12 cells was significant, some of the difference may also be due to the reduction in proliferation.

We next tested whether the observed reduced proliferation and impaired migration of E10 and E12 cells are the result of the increased PHD2 activity and reduced HIF1 $\alpha$ levels. To do this, we treated the cells with the HIF-PHD inhibitor, FG-4592 (roxadustat), or transfected the cells with either HIF1 $\alpha$ or PHD2 expression vectors. Treatment of cells with FG-4592 stabilized HIF1 $\alpha$ and counteracted the reduced proliferation and migration in E10 and E12 cells without having significant effects in control S cells (Supplementary Fig. S7A-S7F). Although overexpression of HIF $1 \alpha$ promoted proliferation, as well as migration in all cells, its action was more pronounced in E10 and E12 cells. Like with FG-4592, this abolished the differences in proliferation and wound healing in E10 cells and largely also in E12 cells

control S cells (hypoxia). F, Quantification of LDHA, GLUT1, CITED2, and VEGF mRNA levels in control S, E10, and E12 cells cultured under normoxia or hypoxia. The respective mRNA levels in control $\mathrm{S}$ cells cultured under normoxia were set to 1 . Data are mean $\pm \mathrm{SD}(n=3){ }^{*}$, significant difference between E10 and E12 cells versus control (normoxia); \#, significant difference between $\mathrm{E} 10$ and $\mathrm{E} 12$ cells versus control $\mathrm{S}$ cells (hypoxia). 

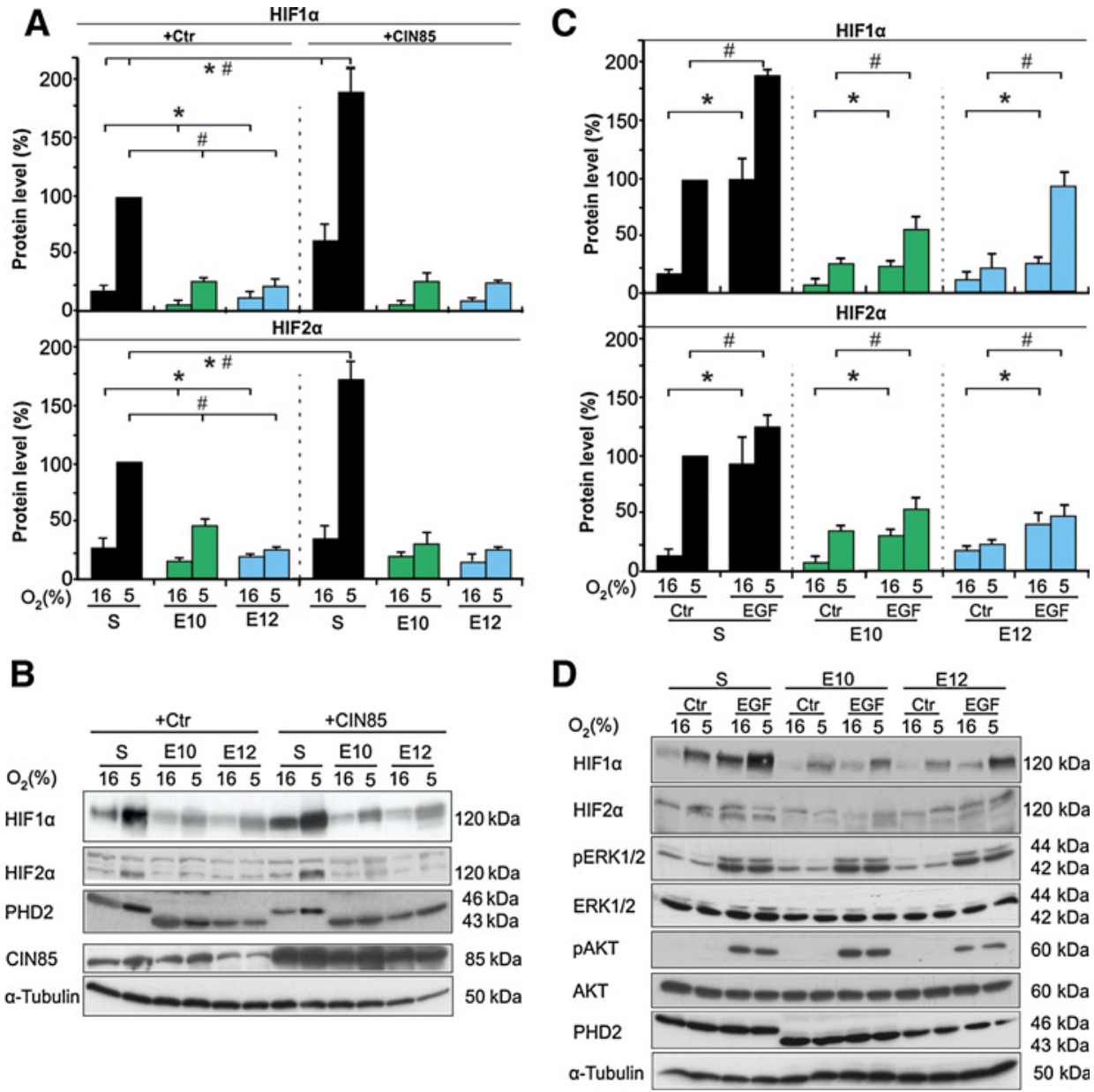

Figure 5.

Lack of the CIN85-PHD2 interaction in MDA-MB-231 cells mediates HIF $\alpha$ resistance to CIN85 overexpression and attenuates EGF-mediated HIF $\alpha$ induction. A, Quantification of HIF1 $\alpha$ and HIF2 $\alpha$ levels in MDA-MB-231 control S, E10, and E12 cells transfected with empty vector ( + Ctr) and in S, E10, and E12 cells with overexpression of CIN85 (+CIN85). Cells were cultured under normoxia $(16 \%)$ or hypoxia $\left(5 \% \mathrm{O}_{2}\right)$ for 4 hours. HIF1 $\alpha$ and HIF2 $\alpha$ levels in S $(+\mathrm{Ctr}$ ) cells under hypoxia were set to $100 \%$. Data are mean $\pm \mathrm{SD}(n=4) ;{ }^{*}$, significant difference between $\mathrm{E} 10$ and $\mathrm{E} 12$ cells versus $\mathrm{S}(+\mathrm{Ctr}$, normoxia); \#, significant difference between $\mathrm{E} 10$ and $\mathrm{E} 12$ cells versus S cells ( $+\mathrm{Ctr}$, hypoxia); ${ }^{\#}$, significant differences S $(+\mathrm{Ctr}$ ) versus $\mathrm{S}(+\mathrm{CIN85})$ under normoxia and hypoxia. B, Western blot analysis. Blots were probed with HIF1 $\alpha$, HIF2 $\alpha$, PHD2, CIN85, and $\alpha$-tubulin antibodies. C, Quantification of HIF1 $\alpha$ and HIF2 $\alpha$ levels in control S, E10, and

E12 cells treated with vehicle (Ctr) or EGF (100 ng/mL). HIFl $\alpha$ and HIF2 $\alpha$ levels in S (+Ctr) cells under hypoxia were set to $100 \%$. Data are mean $\pm \mathrm{SD}(n=3)$; *, significant difference between vehicle (Ctr)-treated S, E10 and E12 cells versus EGF-treated S, E10, and E12 cells (normoxia); \#, significant difference between vehicle (Ctr)-treated S, E10, and E12 cells versus EGF-treated S, E10, and E12 cells (hypoxia). D, Western blot analysis. Blots were probed with antibodies against HIF1 $\alpha$, HIF2 $\alpha$, pERK, total ERK, PAKT, total AKT, PHD2, and tubulin.

(Supplementary Fig. S7G and S7H). In contrast, overexpression of full length PHD2 inhibited proliferation and wound healing significantly only in S cells and marginally but insignificantly in E10 and E12 cells (Supplementary Fig. S7G and S7H). Because the observed changes were not attributable to the off-target effects of the CRISPR/Cas9-mediated EGLN1 gene editing (Supplementary Fig. S8A-S8L), these findings support the view that the observed cellular phenotypes of E10 and E12 cells are largely dependent on both, the activity of PHD2 and HIF1 $\alpha$.

Because the abovementioned assays pointed to a less malignant phenotype of E10 and E12 cells compared with control S cells, we next investigated whether these characteristics are also present in vivo. Therefore, we transplanted MDA-MB-231 control $S$ versus $\mathrm{E} 10$ and $\mathrm{S}$ versus $\mathrm{E} 12$ cells, into the thoracic and inguinal mammary fat pads of 18 female athymic immune-deficient nude mice and followed tumor growth by palpation and size measurement for up to 8 weeks. Although all cells were able to form tumors in these mice, it was obvious that the arisen tumors from control $S$ cells were of bigger volume, compared with tumors formed from E10 and E12 cells (Fig. 6F and G). While the average volume of tumors from control S cells was about $138 \mathrm{~mm}^{3}$ at the time of necropsy, the E10- and E12-derived tumors had a volume of about $36 \mathrm{~mm}^{3}$ and $44 \mathrm{~mm}^{3}$, respectively (Fig. $6 \mathrm{~F}-\mathrm{H}$ ). We also noted that the E10- and E12 cell-derived tumors were paler when compared with the tumors formed from control S cells, which is likely a result from reduced angiogenesis due to lower HIF $\alpha$ levels (Fig. 6F-H).

Collectively, these data show that MDA-MB-231 cells expressing $\triangle 75-103$ PHD2, and thus lacking the interaction between PHD2 and CIN85, are less motile, have a lower proliferative, as well as tumor forming potential. 
Kozlova et al.

A

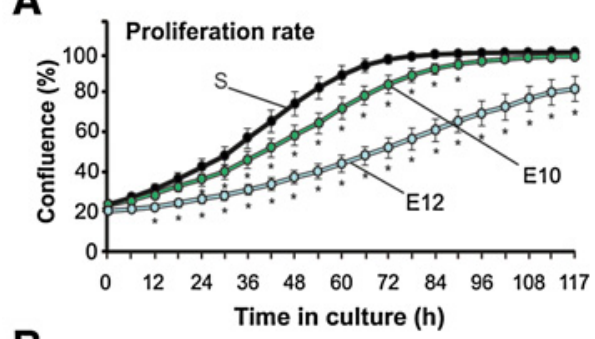

B
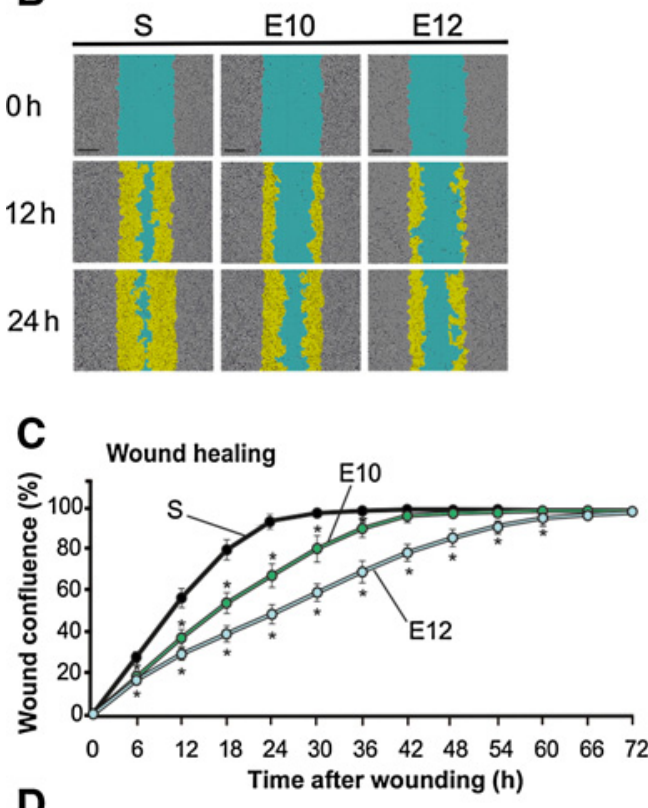

D

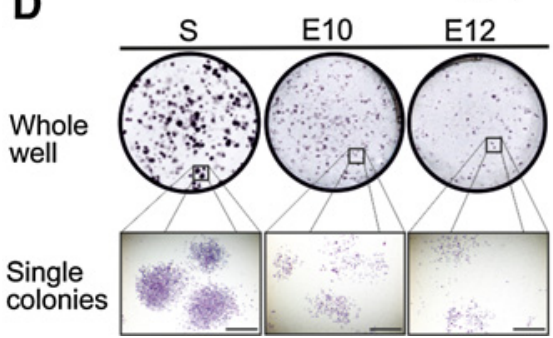

E

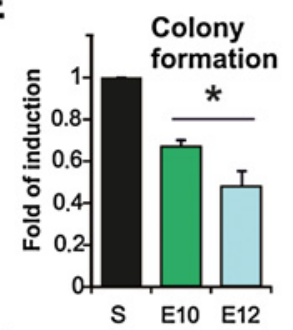

F

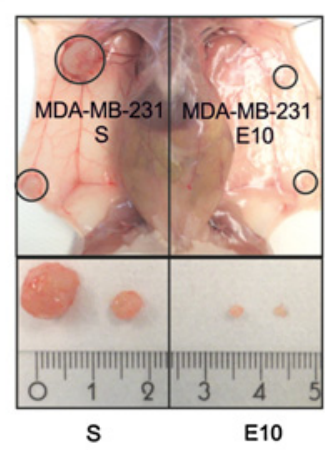

H

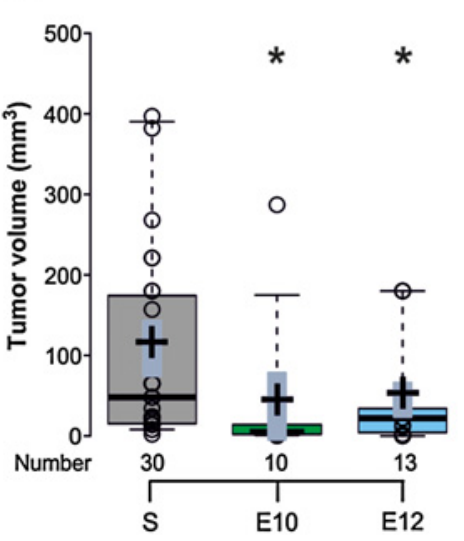

G

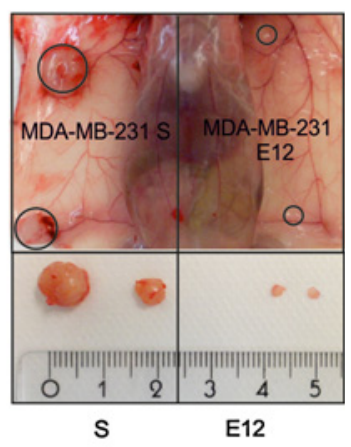

Figure 6.

Lack of amino acids 75-103 in PHD2 reduces malignant properties of MDA-MB-231 cells. A, Live cell proliferation analysis of MDA-MB-231 control S, E10, and E12 cells (see Material and Methods). Data are mean \pm SD $(n=5)$; * significant difference between relative confluence values of $\Delta 75-103$ PHD2-expressing cells (E10 and E12) versus control S cells at each timepoint. B, Representative images of the wound closure of control S, E10, and E12 cells at 0, 12, and 24 hours timepoint after introduction of the wound. Scale bar, $300 \mu \mathrm{m}$. C, Live cell wound closure analysis of MDA-MB-231 S, E10, and E12 cells (see Material and Methods). Data are mean $\pm \mathrm{SD}(n=4) ;{ }^{*}$, significant difference between wound confluence values of $\Delta 75-103$ PHD2-expressing cells (E10 and E12) versus control S cells at each timepoint. D, Representative images of the whole-cell culture wells and single colonies formed by MDA-MB-231 S, E10, and E12 cells. Scale bar, $1 \mathrm{~mm}$. E, Quantification of the colony formation assay. The total number of colonies per well was set to 1 in control S cells. Data are mean $\pm \operatorname{SD}(n=4) ;{ }^{*}$, significant difference between the number of colonies formed by E10 and E12 cells versus control S cells. MDA-MB-231 control S, E10, and E12 cells were injected into the thoracic and inguinal mammary fat pads of female athymic nude mice ( 6 animals per group). $\mathbf{F}$ and $\mathbf{G}$, Representative image of tumors derived from control $\mathbf{S}$ versus E10 (F) and control S versus E12 (G). H, Boxplot indicating the respective volume differences of the control S-derived tumors compared with the E10- and E12-derived tumors. Center lines, medians; crosses, sample means; gray bars within boxes, $83 \%$ confidence intervals of the means; the box limits indicate the 25 th and 75th percentiles as determined by R software; whiskers extend to minimum and maximum values; data points are plotted as open circles. Number $=30,10$, 13 tumor sample points. *, significant difference of the means S versus E10 and E12.

\section{Discussion}

This investigation shows that the prooncogenic adaptor protein, CIN85, is a novel inhibitory binding partner of PHD2 (Fig. 1). We defined the nature of CIN85-PHD2 complex forma- tion by revealing the importance of the three N-terminal SH3 domains of CIN85 and the proline and arginine residues within the $\mathrm{N}$-terminus of PHD2, lying outside of the zinc-finger domain of PHD2 (Figs. 2 and 3). By performing CRISPR/Cas9-mediated EGLN1 gene editing, we created MDA-MB-231 cell lines, 
expressing a PHD2 lacking amino acids 75-103, which was unable to bind CIN85 (Fig. 4). The abrogation of the PHD2CIN85 interaction led to lower HIF1 $\alpha$ and HIF2 $\alpha$ levels (Figs. 4 and 5), thus contributing to a less malignant phenotype of MDAMB-231 cells (Fig. 6).

Interestingly, from three HIF-regulating PHDs, CIN85 binds only PHD2 because PHD2, unlike PHD1 and 3, contains an extended N-terminus with the MYND-zinc-finger part, and as shown here the atypical SH3 domain docking sites. In fact, BLAST results show that PHD2 shares only similarity with the other PHDs in the catalytic domains, where amino acids 192-417 of PHD2 share a $68 \%$ identity with amino acids $176-403$ of the catalytic domain of PHD1. PHD3's amino acids 2-225 share an overall identity of $64 \%$ with PHD2, which resides between amino acids 181-403 of PHD2.

In addition, this study also shows that a close relative of CIN85, CD2AP can be in complex with CIN85 and PHD2 (Fig. 2C); however, the complex formation is facilitated by different interactions between the proteins. It appears that the $\mathrm{SH} 3$ domains of CIN85 and the N-terminus of PHD2 interact, as well as the CC domain of CIN85 with CD2AP. This supports the earlier view that a number of nonredundant functions between CIN85 and CD2AP exist (25). Indeed, CD2AP differs from CIN85 by the presence of additional actin-binding sites before the CC domain (26), and by the lack of one proline-rich segment and the PEST sequence proximal to the CC domain (25). Moreover, a recent publication employing detailed biophysical analyses has shown that, apart from the CC domain, important differences in the orientation of the SH3 domains of CD2AP and CIN85 upon interaction with their partners exist (27). Thus, these differences may account for the observed distinct biological behavior toward PHD2 and imply that the context, in particular that of the binding partner, is important and that there is no single, exact motif in the target molecules binding the SH3 domains. There is rather a core of critical residues in the binding partner, which differ with respect to the type of residues in flanking positions mediating the binding Thus, the sequence context of the binding partner, for example, PHD2 appear to dictate which residues determine its interaction potential. Hence, even if the difference in sequence and structure between the SH3 domains between CIN85 and CD2AP appears to be minimal, the difference in affinity for the binding interface might be rather large. For example, if a Lys (e.g., K114 in CD2AP) is replaced by a charge-neutral amino acid (e.g., Q 104) in CIN85, the structure difference may be minimal, but the difference in affinity for the interface at the binding partner might be rather large. This is underlined by a recent study employing flanking residue permutation arrays in the CD2AP-binding protein, ALIX, where selectivity could be achieved even between the three $\mathrm{SH} 3$ domains (28). Similar effects were also reported for the CIN85 SH3 domains (29). After all, a possibly minimal structural difference can become clearly significant for the observed behavior (binding), but may probably not very much affect another type of behavior (e.g., solubility). Thus, there might be some functional redundancy but also quite a lot of difference between the function of CIN85 and CD2AP where these contexts need to be considered. This is further exemplified by findings from mice and humans, indicating that CIN85 and CD2AP have indeed distinct functions. In particular, a germline deletion within the CIN85 gene on the human X chromosome caused antibody deficiency due to defects in B lymphocyte effector pathways such as NF- $\kappa \mathrm{B}$ and upregulation of CD86 expression (30). In contrast, SNPs or deletions of the
$C D 2 A P$ gene led to abnormal kidney function and proteinuria with nephrotic syndrome caused by focal segmental glomerulosclerosis (31). Overall, these data, in line with this study, suggest that there are a number of nonredundant functions in the action of CIN85 and CD2AP.

The overexpression of CIN85 is known to influence the development and progression of various types of cancers, among which are carcinomas of the head and neck (32), cervix (33), and colon (12). Our own research showed that CIN85 is highly overexpressed in breast carcinoma (11), and contributes to breast cancer pathogenesis via the attenuation of EGFR and ErbB2/HER2 downregulation $(11,34)$. In addition, the mechanisms behind CIN85-driven carcinogenesis include an enhancement of TGF- $\beta$ signaling and promotion of cell invasiveness via focal adhesion kinase (35). Moreover, our previous findings indicated that CIN85 contributes to cancer progression via stabilization of HIF1/2 $\alpha$ (as well as the expression of HIF target genes such as PAI-1; ref. 13). This study extended these findings and verified that the direct interaction of CIN85 and PHD2 can inhibit PHD2mediated prolyl-hydroxylation of HIF $\alpha$ subunits.

The HIF family of transcription factors are essential molecular regulators of normal development and at the same time serve as crucial contributors to the progression of various types of cancers (36). Although HIF $\alpha$ levels are regulated via multiple mechanisms (3), the hydroxylation-dependent mechanism is considered to have a major impact on HIF1 $\alpha$ degradation. All members of the HIF-PHD family are able to hydroxylate HIF1 $\alpha$ in vitro (37); however, PHD2 is found to be the most important hydroxylase to regulate HIF1 $\alpha$ levels under normoxic conditions $(20,24)$. The activity of PHD2 is critical for HIF $\alpha$ stability; however, alterations in the enzymatic function of PHD2 are not always caused by the lack of oxygen (38). The enzymatic function of PHD2 can be indirectly altered through the association with other proteins that interfere with the PHD2/HIFos/VHL complex formation.

The majority of PHD2 interactors contribute to the downregulation of HIF $\alpha$ levels. For example, inhibitor of growth family member 4 (ING4; ref. 39), p23 from the HSP90 machinery (40), and the runt-domain transcription factor (41) were reported to promote the association between PHD2 and HIF $\alpha$ that leads to a faster downregulation of HIF1 $\alpha$. Another example is the direct interaction of the adaptor proteins LIMD1 and RHO-related BTB domain-containing protein 3 (RHOBTB3) with PHD2 and VHL simultaneously (42). The association of PHD2 with these adaptors promotes the assembly of hydroxylation-dependent degradation machinery, which further contributes to lower levels of both HIF $1 \alpha$ and HIF2 $\alpha$ (42). Because decreased levels of HIF $\alpha$ s are usually considered to favor patient survival, the participation of RUNX3 (43), ING4 (44), LIMD1 (45), and RHOBTB3 (46) in downregulating HIFos additionally confirms the known tumorsuppressing role of these proteins in human cancers. Interestingly, our data show that the strength of the interaction between PHD2 and CIN85 varies between the cell lines investigated. This could suggest that (an)other factor(s), existence of which could vary between the different cell types, may contribute to the strength of the interaction and future studies will show whether this is the case.

The results of our study indicate that the interaction of CIN85 with PHD2 leads to a very different outcome by contributing to the increased stability of HIF1 $\alpha$ and HIF2 $\alpha$. Our current investigation describes a direct interaction with PHD2 as a mechanism 
by which CIN85 regulates HIF1 $\alpha$ and HIF2 $\alpha$ stability in breast cancer cells. This event appears to occur in the cytoplasm because we found that the CIN85-PHD2 interaction was predominantly cytoplasmic (Fig. 1). This is in line with reports that, both PHD2 and CIN85 are cytoplasmic proteins although PHD2 has also been found in the nucleus (47). In addition, we showed that CIN85 utilizes three SH3 domains to facilitate the binding to PHD2 (Fig. 2).

While the majority of SH3 domains bind "typical" proline-rich motifs like PXXP (where X is any amino acid), the SH3 domains of CIN85 are reported to recognize atypical PR sequences (23). By using a variety of PHD2 and CIN85 constructs in immunoprecipitation and GST-pull down experiments, we found that a PR-rich area between amino acids $77-100$ is the most important for the direct interaction with CIN85 (Figs. 1-3).

While PHD2-binding partners are known to utilize either the Zn-MYND finger domain (48), or the catalytic domain of PHD2, we define CIN85 as the first interactor recognizing a different binding motif in the sequence of PHD2 (Fig. 3). The mapping of the binding motif for CIN85 not only identified critical residues mediating the CIN85-PHD2 complex formation, but also revealed the existence of a functional sequence in the $\mathrm{N}$-terminus of PHD2 able to mediate protein-protein interactions. Although this approach was straight forward, there exists also the possibility that the systematic mutation of the proline residues may have led to a conformational change in PHD2 in other regions, thus influencing the binding between CIN85 and PHD2 indirectly. Interestingly, the PHD2 variants lacking the ability to interact with CIN85 showed higher catalytic activity in the in vitro hydroxylation assay, thus leading to lower levels of HIF1 $\alpha$. Although the structural basis for oxygen degradation domain selectivity of the catalytic PHD regions are quite well known (49), the crystal structural details of the PHD2 N-terminus harboring the CIN85 interaction site remain so far unresolved. However, the current data are in line with earlier findings showing that the $\mathrm{N}$-terminus of PHD2 is inhibitory (50). This, in line with the in vitro hydroxylation assays of this study, suggests that binding of CIN85 might regulate PHD2 activity at the $\mathrm{N}$-terminus by reducing the accessibility of its catalytic domain to a substrate.

After establishing the binding pattern between CIN85 and PHD2, we created a cell system lacking this molecular interplay using CRISPR/Cas9-mediated EGLN1 gene editing. Two independent cell clones expressing PHD2-lacking amino acids 75-103 and consequently the interaction between CIN85 and PHD2 were generated (Fig. 4). The MDA-MB-231 $\Delta 75-103$ PHD2-expressing cell lines E10 and E12 showed, as expected, lower HIF1 $\alpha$ and HIF $2 \alpha$ protein levels (Fig. 5), and slower proliferation, decreased motility, as well as a reduced tumor forming potential in nude mice when compared with the respective control cells (Fig. 6).

Our previous findings indicated that overexpression of CIN85 stabilizes HIF1 $\alpha$ levels, and notably, that downregulation of CIN85 reversed this effect (13). The results of this study allow

\section{References}

1. Laughner E, Taghavi P, Chiles K, Mahon PC, Semenza GL. HER2 (neu) signaling increases the rate of hypoxia-inducible factor 1alpha (HIF-1alpha) synthesis: Novel mechanism for HIF-1-mediated vascular endothelial growth factor expression. Mol Cell Biol 2001;21: 3995-4004.

2. Semenza GL. Molecular mechanisms mediating metastasis of hypoxic breast cancer cells. Trends Mol Med 2012;18:534-43. us to conclude that lower HIF1 $\alpha$ levels under normoxic conditions in $\triangle 75-103$ PHD2-expressing cells originate from decreased protein stability and cannot be attributed to the differences in HIF $\alpha$ mRNA expression. In addition, we can associate the observed downregulation in HIF1 $\alpha$ and HIF $2 \alpha$ levels with 475-103 PHD2 function, because the expression of the two other PHDs (PHD1 and PHD3) was not affected (Supplementary Fig. S4A-S4I).

Altogether, our study is the first to describe the relation between PHD2 and CIN85. We identified CIN85 as a novel inhibitory binding partner of PHD2, able to increase HIF $\alpha$ levels and, thus, to promote HIF $\alpha$ stability and breast cancer malignancy.

\section{Disclosure of Potential Conflicts of Interest}

No potential conflicts of interest were disclosed.

\section{Authors' Contributions}

Conception and design: N. Kozlova, L.B. Drobot, T. Kietzmann Development of methodology: N. Kozlova, E.Y. Dimova, E. Biterova A. Hassinen, A. Manninen, L.B. Drobot, T. Kietzmann

Acquisition of data (provided animals, acquired and managed patients, provided facilities, etc.): N. Kozlova, A. Samoylenko, P. Koivunen, E. Biterova, K. Richter, A. Hassinen, I. Miinalainen, V. Glumoff, T. Kietzmann Analysis and interpretation of data (e.g., statistical analysis, biostatistics, computational analysis): N. Kozlova, A. Samoylenko, I. Miinalainen, L. Ruddock, T. Kietzmann

Writing, review, and/or revision of the manuscript: N. Kozlova, D. Mennerich, A. Samoylenko, S. Kellokumpu, I. Miinalainen, L. Ruddock, L.B. Drobot, T. Kietzmann

Administrative, technical, or material support (i.e., reporting or organizing data, constructing databases): N. Kozlova, S. Kellokumpu, A. Manninen, T. Kietzmann

Study supervision: T. Kietzmann

\section{Acknowledgments}

The authors are grateful to Dr. Svetlana Marchenko for help with the GST pull-down experiments in the beginning of the study, Dr. Kristian Koski for help with protein purification, Jaana Träskelin and Dr. Satu Myllymäki for help with the production of lentiviral particles, and Lea Boten, Jonas Böhm, Lea Cleve, Anabel Arnemann (School of Life Science Hamburg, Germany), and Maire Jarva for excellent technical assistance. The work in the authors' laboratories was supported by grants from the Federation of European Biochemical Societies (to N. Kozlova), Finnish Center of International Mobility (to T. Kietzmann), Finnish Academy of Sciences (SA296027 to T. Kietzmann and SA308009 to P. Koivunen), Jane and Aatos Erkko Foundation (to T. Kietzmann and P. Koivunen), Finnish Cancer Foundation (to T. Kietzmann and P. Koivunen), Sigrid Juselius Foundation (to T. Kietzmann, P. Koivunen, and L. Ruddock), Biocenter Oulu (to T. Kietzmann, P. Koivunen, A. Manninen, I. Miinalainen, and L. Ruddock), and University of Oulu supported this work.

The costs of publication of this article were defrayed in part by the payment of page charges. This article must therefore be hereby marked advertisement in accordance with 18 U.S.C. Section 1734 solely to indicate this fact.

Received December 11, 2018; revised April 17, 2019; accepted May 24, 2019; published first May 29, 2019

3. Flugel D, Gorlach A, Kietzmann T. GSK-3beta regulates cell growth, migration, and angiogenesis via Fbw7 and USP28-dependent degradation of HIF-1alpha. Blood 2012;119:1292-301.

4. Ivan M, Haberberger T, Gervasi DC, Michelson KS, Gunzler V, Kondo K, et al. Biochemical purification and pharmacological inhibition of a mammalian prolyl hydroxylase acting on hypoxia-inducible factor. Proc Nat Acad Sci U S A 2002;99:13459-64. 
5. Jaakkola P, Mole DR, Tian YM, Wilson MI, Gielbert J, Gaskell SJ, et al Targeting of HIF-alpha to the von hippel-lindau ubiquitylation complex by O2-regulated prolyl hydroxylation. Science 2001;292:468-72.

6. Soubeyran P, Kowanetz K, Szymkiewicz I, Langdon WY, Dikic I. CblCIN85-endophilin complex mediates ligand-induced downregulation of EGF receptors. Nature 2002;416:183-7.

7. Minegishi Y, Shibagaki Y, Mizutani A, Fujita K, Tezuka T, Kinoshita M, et al. Adaptor protein complex of FRS2ß and CIN85/CD2AP provides a novel mechanism for ErbB2/HER2 protein downregulation. Cancer Sci 2013; 104:345-52.

8. Petrelli A, Gilestro GF, Lanzardo S, Comoglio PM, Migone N, Giordano S. The endophilin-CIN85-cbl complex mediates ligand-dependent downregulation of c-met. Nature 2002;416:187-90.

9. Havrylov S, Redowicz MJ, Buchman VL. Emerging roles of Ruk/CIN85 in vesicle-mediated transport, adhesion, migration and malignancy. Traffic 2010;11:721-31.

10. Nam JM, Onodera Y, Mazaki Y, Miyoshi H, Hashimoto S, Sabe H. CIN85, a cbl-interacting protein, is a component of AMAP1-mediated breast cancer invasion machinery. EMBO J 2007;26:647-56.

11. Samoylenko A, Vynnytska-Myronovska B, Byts N, Kozlova N, Basaraba O, Pasichnyk $\mathrm{G}$, et al. Increased levels of the HER1 adaptor protein Rukl/ CIN85 contribute to breast cancer malignancy. Carcinogenesis 2012;33: 1976-84.

12. Cascio S, Finn OJ. Complex of MUC1, CIN85 and cbl in colon cancer progression and metastasis. Cancers 2015;7:342-52.

13. Samoylenko A, Dimova EY, Kozlova N, Drobot L, Kietzmann T. The adaptor protein Ruk/CIN85 activates plasminogen activator inhibitor-1 (PAI-1) expression via hypoxia-inducible factor-1alpha. Thromb Haemost 2010;103:901-9.

14. Kerppola TK. Design and implementation of bimolecular fluorescence complementation (BiFC) assays for the visualization of protein interactions in living cells. Nat Protoc 2006;1:1278-86.

15. Gout I, Middleton G, Adu J, Ninkina NN, Drobot LB, Filonenko V, et al. Negative regulation of PI 3-kinase by Ruk, a novel adaptor protein. EMBO J 2000;19:4015-25.

16. Scharf JG, Unterman TG, Kietzmann T. Oxygen-dependent modulation of insulin-like growth factor binding protein biosynthesis in primary cultures of rat hepatocytes. Endocrinology 2005;146:5433-43.

17. Mayevska O, Shuvayeva H, Igumentseva N, Havrylov S, Basaraba O, Bobak Y, et al. Expression of adaptor protein Ruk/CIN85 isoforms in cell lines of various tissue origins and human melanoma. Exp Oncol 2006;28:275-81.

18. Hirsila M, Koivunen P, Gunzler V, Kivirikko KI, Myllyharju J. Characterization of the human prolyl 4-hydroxylases that modify the hypoxiainducible factor. J Biol Chem 2003;278:30772-80.

19. Konzack A, Jakupovic M, Kubaichuk K, Gorlach A, Dombrowski F, Miinalainen I, et al. Mitochondrial dysfunction due to lack of manganese superoxide dismutase promotes hepatocarcinogenesis. Antioxid Redox Signal 2015;23:1059-75.

20. Berra E, Benizri E, Ginouves A, Volmat V, Roux D, Pouyssegur J. HIF prolylhydroxylase 2 is the key oxygen sensor setting low steady-state levels of HIF1alpha in normoxia. EMBO J 2003;22:4082-90.

21. Kirsch KH, Georgescu MM, Shishido T, Langdon WY, Birge RB, Hanafusa H. The adapter type protein CMS/CD2AP binds to the proto-oncogenic protein c-cbl through a tyrosine phosphorylation-regulated Src homology 3 domain interaction. J Biol Chem 2001;276:4957-63.

22. Dikic I. CIN85/CMS family of adaptor molecules. FEBS Lett 2002;529: 110-5.

23. Kurakin AV, Wu S, Bredesen DE. Atypical recognition consensus of CIN85/ SETA/Ruk SH3 domains revealed by target-assisted iterative screening. J Biol Chem 2003;278:34102-9.

24. Appelhoff RJ, Tian YM, Raval RR, Turley H, Harris AL, Pugh CW, et al. Differential function of the prolyl hydroxylases PHD1, PHD2, and PHD3 in the regulation of hypoxia-inducible factor. J Biol Chem 2004;279: 38458-65.

25. Tibaldi EV, Reinherz EL. CD2BP3, CIN 85 and the structurally related adaptor protein CMS bind to the same CD2 cytoplasmic segment, but elicit divergent functional activities. Int Immunol 2003;15:313-29.

26. Gaidos G, Soni S, Oswald DJ, Toselli PA, Kirsch KH. Structure and function analysis of the CMS/CIN85 protein family identifies actin- bundling properties and heterotypic-complex formation. J Cell Sci 2007;120:2366-77.

27. Ceregido MA, Garcia-Pino A, Ortega-Roldan JL, Casares S, Lopez Mayorga $\mathrm{O}$, Bravo J, et al. Multimeric and differential binding of CIN85/CD2AP with two atypical proline-rich sequences from CD2 and cbl-b*. FEBS J 2013;280: 3399-415.

28. Rouka E, Simister PC, Janning M, Kumbrink J, Konstantinou T, Muniz JR, et al. Differential recognition preferences of the three Src homology 3 (SH3) domains from the adaptor CD2-associated protein (CD2AP) and direct association with ras and rab interactor 3 (RIN3). J Biol Chem 2015; 290:25275-92.

29. Ababou A, Pfuhl M, Ladbury JE. Novel insights into the mechanisms of CIN85 SH3 domains binding to Cbl proteins: Solution-based investigations and in vivo implications. J Mol Biol 2009;387:1120-36.

30. Keller B, Shoukier M, Schulz K, Bhatt A, Heine I, Strohmeier V, et al Germline deletion of CIN85 in humans with X chromosome-linked antibody deficiency. J Exp Med 2018;215:1327-36.

31. Takano T, Bareke E, Takeda N, Aoudjit L, Baldwin C, Pisano P, et al. Recessive mutation in CD2AP causes focal segmental glomerulosclerosis in humans and mice. Kidney Int 2019;95:57-61.

32. Wakasaki $T$, Masuda M, Niiro H, Jabbarzadeh-Tabrizi S, Noda K, Taniyama $\mathrm{T}$, et al. A critical role of c-Cbl-interacting protein of $85 \mathrm{kDa}$ in the development and progression of head and neck squamous cell carcinomas through the ras-ERK pathway. Neoplasia 2010;12:789-96.

33. Ma Y, Ye F, Xie X, Zhou C, Lu W. Significance of PTPRZ1 and CIN85 expression in cervical carcinoma. Arch Gynecol Obstet 2011;284: 699-704.

34. Schroeder B, Weller SG, Chen J, Billadeau D, McNiven MA. A Dyn2-CIN85 complex mediates degradative traffic of the EGFR by regulation of late endosomal budding. EMBO J 2010;29:3039-53.

35. Schmidt MHH, Chen B, Randazzo LM, Bögler O. SETA/CIN85/Ruk and its binding partner AIP1 associate with diverse cytoskeletal elements, including FAKs, and modulate cell adhesion. J Cell Sci 2003;116: 2845-55.

36. Dales JP, Garcia S, Meunier-Carpentier S, Andrac-Meyer L, Haddad O, Lavaut $\mathrm{MN}$, et al. Overexpression of hypoxia-inducible factor HIF-1a predicts early relapse in breast cancer: Retrospective study in a series of 745 patients. Int J Cancer 2005;116:734-9.

37. Koivunen P, Tiainen P, Hyvarinen J, Williams KE, Sormunen R, Klaus SJ, et al. An endoplasmic reticulum transmembrane prolyl 4-hydroxylase is induced by hypoxia and acts on hypoxia-inducible factor alpha. J Biol Chem 2007;282:30544-52.

38. Stolze IP, Mole DR, Ratcliffe PJ. Regulation of HIF: prolyl hydroxylases. Novartis Found Symp 2006;272:15-25.

39. Colla S, Tagliaferri S, Morandi F, Lunghi P, Donofrio G, Martorana D, et al. The new tumor-suppressor gene inhibitor of growth family member 4 (ING4) regulates the production of proangiogenic molecules by myeloma cells and suppresses hypoxia-inducible factor- 1 alpha (HIF-1alpha) activity: involvement in myeloma-induced angiogenesis. Blood 2007;110: 4464-75.

40. Song D, Li LS, Heaton-Johnson KJ, Arsenault PR, Master SR, Lee FS. Prolyl hydroxylase domain protein 2 (PHD2) binds a Pro-Xaa-Leu-Glu motif, linking it to the heat shock protein 90 pathway. J Biol Chem 2013;288: 9662-74.

41. Lee SH, Bae SC, Kim KW, Lee YM. RUNX3 inhibits hypoxia-inducible factor-1 a protein stability by interacting with prolyl hydroxylases in gastric cancer cells. Oncogene 2014;33:1458-67.

42. Foxler DE, Bridge KS, James V, Webb TM, Mee M, Wong SC, et al. The LIMD1 protein bridges an association between the prolyl hydroxylases and VHL to repress HIF-1 activity. Nat Cell Biol 2012;14:201-8.

43. Yu YY, Chen C, Kong F-, Zhang W. Clinicopathological significance and potential drug target of RUNX3 in breast cancer. Drug Des DevTher 2014;8: 2423-30.

44. Ozer A, Bruick RK. Regulation of HIF by prolyl hydroxylases: recruitment of the candidate tumor suppressor protein ING4. Cell Cycle 2005; 4:1153-6.

45. Sharp TV, Munoz F, Bourboulia D, Presneau N, Darai E, Wang HW, et al. LIM domains-containing protein 1 (LIMD1), a tumor suppressor encoded at chromosome 3 p21.3, binds pRB and represses E2F-driven transcription. Proc Natl Acad Sci U S A 2004;101:16531-6. 
Kozlova et al.

46. Zhang CS, Liu Q, Li M, Lin SY, Peng Y, Wu D, et al. RHOBTB3 promotes proteasomal degradation of HIF $\alpha$ through facilitating hydroxylation and suppresses the Warburg effect. Cell Res 2015;25:1025-42.

47. Pientka FK, Hu J, Schindler SG, Brix B, Thiel A, Jöhren O, et al. Oxygen sensing by the prolyl-4-hydroxylase PHD2 within the nuclear compartment and the influence of compartmentalisation on HIF-1 signalling. J Cell Sci 2012;125:5168-76.

48. Barth S, Edlich F, Berchner-Pfannschmidt U, Gneuss S, Jahreis G, Hasgall PA, et al. Hypoxia-inducible factor prolyl-4-hydroxylase PHD2 protein abundance depends on integral membrane anchoring of FKBP38. J Biol Chem 2009;284:23046-58.

49. Chowdhury R, Leung IK, Tian YM, Abboud MI, Ge W, Domene C, et al. Structural basis for oxygen degradation domain selectivity of the HIF prolyl hydroxylases. Nat Commun 2016;7:12673.

50. Choi KO, Lee $\mathrm{T}$, Lee $\mathrm{N}$, Kim JH, Yang EG, Yoon JM, et al. Inhibition of the catalytic activity of hypoxia-inducible factor-1alpha-prolylhydroxylase 2 by a MYND-type zinc finger. Mol Pharmacol 2005;68: 1803-9. 


\section{Cancer Research}

\section{The Pro-Oncogenic Adaptor CIN85 Acts as an Inhibitory Binding Partner of Hypoxia-Inducible Factor Prolyl Hydroxylase 2}

Nina Kozlova, Daniela Mennerich, Anatoly Samoylenko, et al.

Cancer Res 2019;79:4042-4056. Published OnlineFirst May 29, 2019.

\section{Updated version Access the most recent version of this article at: doi:10.1158/0008-5472.CAN-18-3852}

Supplementary Access the most recent supplemental material at:

Material http://cancerres.aacrjournals.org/content/suppl/2019/05/29/0008-5472.CAN-18-3852.DC1

Visual A diagrammatic summary of the major findings and biological implications:

Overview http://cancerres.aacrjournals.org/content/79/16/4042/F1.large.jpg

Cited articles This article cites 50 articles, 23 of which you can access for free at:

http://cancerres.aacrjournals.org/content/79/16/4042.full\#ref-list-1

E-mail alerts Sign up to receive free email-alerts related to this article or journal.

Reprints and To order reprints of this article or to subscribe to the journal, contact the AACR Publications Department a Subscriptions pubs@aacr.org.

Permissions To request permission to re-use all or part of this article, use this link http://cancerres.aacrjournals.org/content/79/16/4042.

Click on "Request Permissions" which will take you to the Copyright Clearance Center's (CCC)

Rightslink site. 